Bulletin de la Société Royale des Sciences de Liège, Vol. 86, articles, 2017, p. 169 - 201

\title{
Minéralogie de l'ancienne mine de plomb de Masbourg
}

(Nassogne, Province de Luxembourg, Belgique)

Manuscrit reçu le 9 octobre 2017 et accepté le 25 octobre 2017

Michel BlondieAu ${ }^{1}$, Stéphane PuCCIO ${ }^{2}$, Frédéric HATERT ${ }^{3}$

${ }^{1}$ Val des Cloches 131, B-6927 Tellin, Belgique

${ }^{2}$ Rue des Fontaines 156, B-4041 Vottem, Belgique

${ }^{3}$ Université de Liège, Laboratoire de Minéralogie B18, B-4000 Liège, Belgique

\section{Résumé}

Le petit filon plombifère de Masbourg est caractérisé par une gangue siliceuse, ce qui est assez typique des petits gisements ardennais mais fort rare en Belgique où la majorité des gisements $\mathrm{Pb}-\mathrm{Zn}$ sont logés dans des encaissants carbonatés. De plus, la chalcopyrite y est localement abondante, ce qui a permis l'apparition d'espèces minérales peu courantes dont deux sont nouvelles pour la Belgique : la calédonite et la susannite.

Mots clés : Minéralogie, mine de plomb, Masbourg, Belgique.

\begin{abstract}
The lead deposit of Masbourg is characterized by a siliceous breccia containing grains of chalcopyrite and galena. This type of deposit is relatively rare in Belgium, where the majority of $\mathrm{Pb}-\mathrm{Zn}$ veins occur in limestones. The abundance of chalcopyrite produced unusual mineral species for Belgium, including caledonite and susannite which are reported here for the first time in our country.
\end{abstract}

Keywords: Mineralogy, lead deposit, Masbourg, Belgium.

\section{Introduction}

À environ $3 \mathrm{~km}$ au sud-est de Masbourg (Province de Luxembourg), au lieu-dit "Le Beoly", on peut encore observer dans un petit bois de feuillus se trouvant près de la route N.849 reliant Forrières à Saint-Hubert (Coordonnées Lambert 2008 : 719224, 587422) (Figure 1) les stigmates des anciens travaux miniers réalisés dans la concession de plomb de Masbourg. Plusieurs trous peu profonds en entonnoirs, autour desquels se trouvent quelques déblais, marquent l'emplacement des anciens puits de mine (Figures 2A et 2B). L'entrée de la galerie, sans doute 
Bulletin de la Société Royale des Sciences de Liège, Vol. 86, articles, 2017, p. 169 - 201

effondrée, était située à l'ouest de la route, aujourd'hui dans l'enceinte du Musée de la Vie rurale en Wallonie.

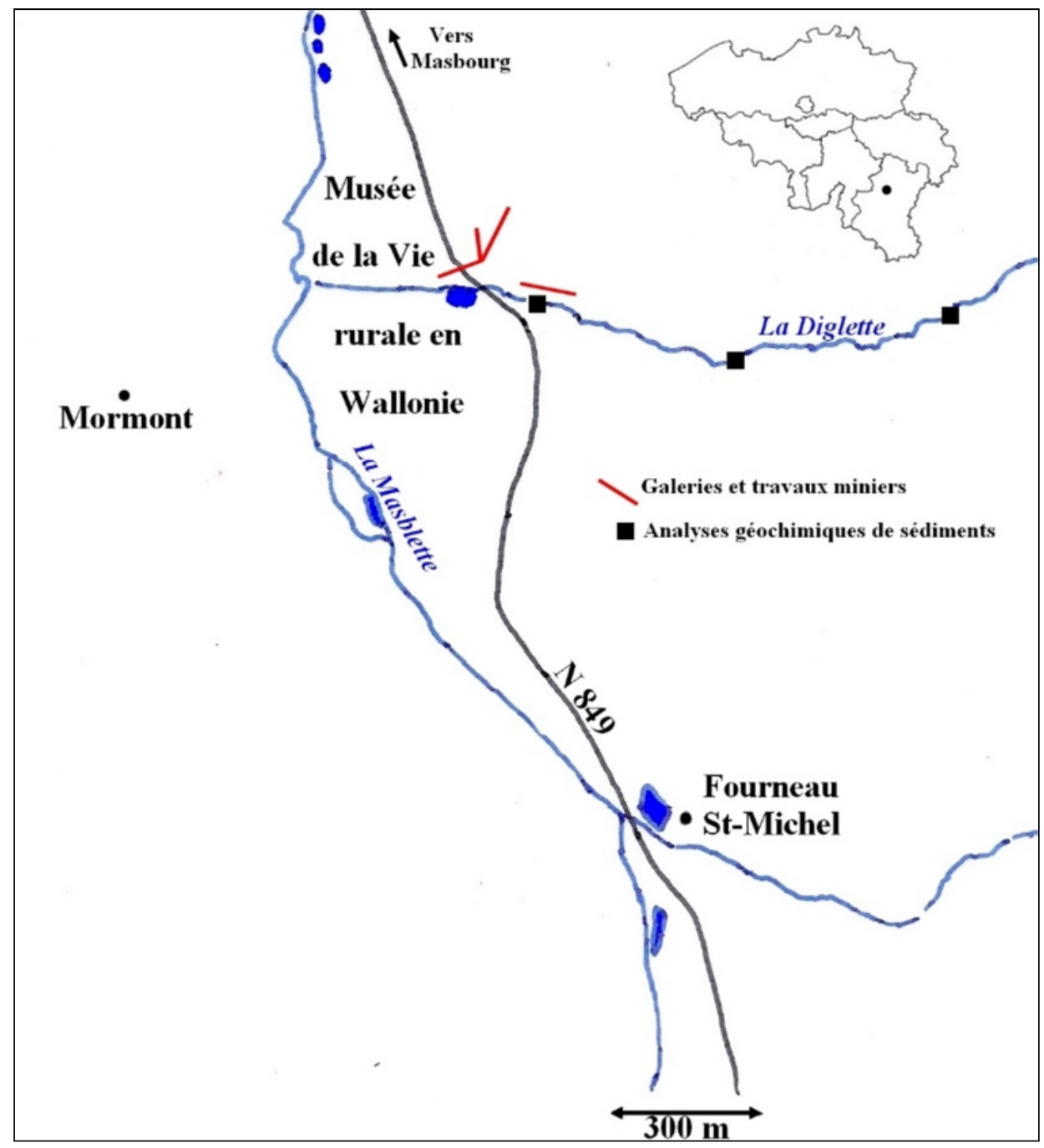

Fig. 1 : Localisation des travaux miniers de Masbourg et des zones de prélèvement d'échantillons de sédiments le long de la Diglette pour analyses géochimiques. En médaillon, localisation de Masbourg sur une carte de Belgique. 

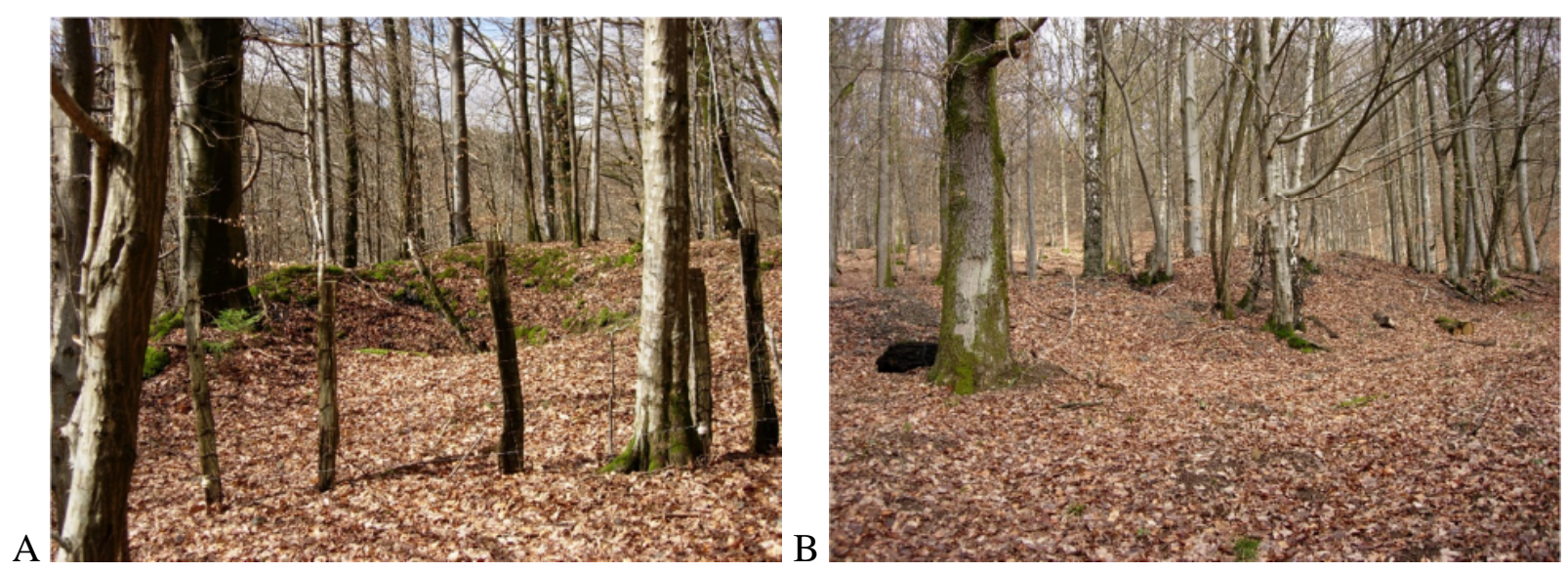

Fig. 2 : Quelques témoins des travaux miniers de Masbourg dans un environnement boisé (Avril 2006). (A) Fosse en entonnoir marquant l'emplacement d'un ancien puits de mine. (B) Déblais miniers dans lesquels ont été récoltés les échantillons étudiés dans cet article.

Bockholz (1921) reprend des indications fournies en 1881 par l'Ingénieur Principal des Mines Ch. Clément qui écrivait :

«Une galerie au niveau du ruisseau de la Diglette explora sur 106 mètres de longueur un filon dirigé Nord-Est et encaissé dans les roches ahrienne (= Emsien inférieur). Des puits de 25 à 30 mètres le mirent en communication avec la surface du sol. D'autres sous-bures servirent à explorer sous la voie d'écoulement des eaux. Ces explorations peu étendues mirent à découvert un gite épais de 0,04 m en moyenne, avec étreintes nombreuses, ou parties privées de minerai, duquel on obtint 53 tonnes de galène. »

Dans ce même document, il ajoute que les travaux ont cessé dès l'obtention de la concession (22 juin 1854). Ceci n'est sans doute pas tout à fait exact, car deux documents signés par l'ingénieur des Mines Poncelet et conservés aux archives de l'état à Arlon, indiquent une certaine activité en 1855 et en 1860, mais ces deux années sont néanmoins déficitaires. Le premier document, signé du 28 avril 1856, indique la présence de 16 ouvriers et une production de 8,5 tonnes de galène. Le second document, signé du 8 mars 1861, mentionne la présence de 9 ouvriers mais sans aucune production de minerai.

Enfin, signalons que lors d'une campagne d'analyses géochimiques de sédiments prélevés dans les rivières de Wallonie (Sonnet, 2017), trois échantillons ont été récoltés dans la Diglette, en amont des anciens travaux miniers. Ces échantillons n'ont cependant pas présenté d'anomalies géochimiques particulières, indiquant sans doute le caractère très confiné des minéralisations. Les galeries et travaux miniers réalisés et les lieux de collectes des échantillons pour analyses géochimiques sont reportés à la Figure 1. 
Bulletin de la Société Royale des Sciences de Liège, Vol. 86, articles, 2017, p. 169 - 201

\section{Contexte géologique}

Blockmans \& Dumoulin (2017) indiquent, sur la version provisoire de la nouvelle carte géologique de Wallonie, que les terrains traversés par les filons de Masbourg appartiennent à la formation de Villé (VIL). Cette formation du dévonien inférieur se trouve dans l'étage Praguien moyen, appelé "Coblencien (Cba)" sur les cartes géologiques du début $\mathrm{XX}^{\mathrm{e}}$ siècle et "Siegenien moyen (S2)" par Asselberghs (1946).

La coupe-type de cette formation se trouve le long de la route de La Roche-en-Ardenne à Houffalize et est décrite par Dejonghe \& Hance (2001) dans une notice de la carte géologique de Wallonie. Selon ces auteurs, cette formation se caractérise par des alternances de trois lithologies:

- Des phyllades bleu sombre à lamines gréseuses rouille.

- Des siltites et grès argileux gris ou bleu avec altération superficielle brun rouille.

- Des grès bleus carbonatés souvent très fossilifères.

L'abondance de calcaire dans certains bancs est typique de cette formation du dévonien inférieur ardennais, lequel en est généralement exempt. D'autres petits filons plombifères ardennais sont également logés dans cette formation. Citons notamment celui de La Roche-en Ardenne (Blondieau \& Hatert, 2009) et celui d'Hoursinne.

Seeliger (1852), sur le plan annexé à la demande en concession, indique deux filons de plomb à Masbourg mais le filon parallèle à la rivière (Figure 1) n'a fait, apparemment, l'objet que de petits travaux de reconnaissance. Sur base des échantillons récoltés, il semble que les failles devaient être fort peu ouvertes, et ont été colmatées principalement par une brèche (Figure 3), constituée d'éléments anguleux de quartzite cimentés par du quartz laiteux dans lequel se développent de manière très irrégulière des cristaux de galène, ainsi que des mouchetures de chalcopyrite.

\section{Descriptions minéralogiques}

Les échantillons étudiés proviennent de fouilles que nous avons réalisées il y a plus de 25 ans dans un petit déblai juste à côté d'un ancien puits de mine (Figure 2B). Les minéraux ont été soumis à une analyse par diffraction des rayons X en mode "Debye-Scherrer », sur un diffractomètre à 4 cercles Rigaku Xcalibur, équipé d'un détecteur EOS et utilisant la radiation $\operatorname{MoK}_{\alpha}(\lambda=0,7107 \AA)$. La morphologie des minéraux a été observée à l'aide d'un microscope électronique à balayage environnemental FEI ESEM-FEG XL 30 (CAREM-ULg, Cellule d'Appui à la Recherche et à l'Enseignement en Microscopie de l'Université de Liège). Les images ont été obtenues en électrons secondaires (mode «haut-vide»), sous une tension d'accélération de $15 \mathrm{kV}$, et sur des échantillons métallisés au platine. Des spectres EDS («Energy Dispersive X-ray Spectrometry») de microanalyse élémentaire aux rayons X ont été obtenus avec le même microscope, équipé d'un détecteur de rayons X Si(Li) EDAX ou SDD 
Bruker $129 \mathrm{eV}$. Selon les cas, la tension d'accélération était réglée à 15, 20 ou $30 \mathrm{kV}$. Les spectres ont été traités soit par le logiciel EDAX, soit par le logiciel Quanta Esprit 1.9 qui a fourni l'identification des éléments et des analyses semi-quantitatives basées sur la matrice de correction ZAF sans standard. Toutes les photographies au microscope électronique à balayage sont prises en mode électrons secondaires.

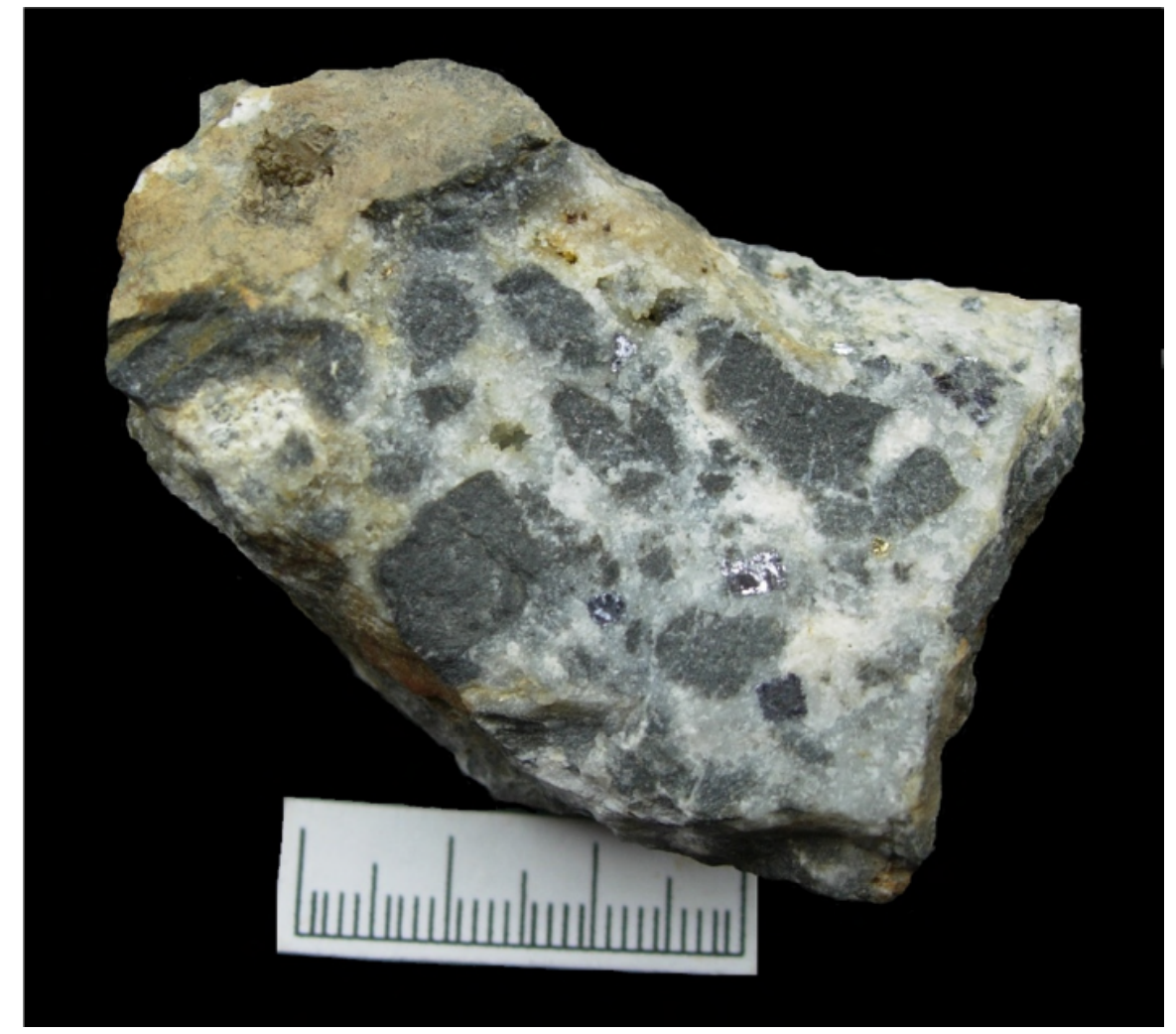

Fig. 3 : Fragment de la brèche filonienne de Masbourg. Des morceaux anguleux de roche sont cimentés par du quartz blanc dans lequel on observe des mouchetures de galène et de chalcopyrite.

\subsection{Anglésite, $\mathrm{PbSO}_{4}$}

L'anglésite est confirmée par diffraction des rayons X. Elle se présente en cristaux adoptant différents habitus cristallins (Figure 4), pouvant atteindre une longueur de plusieurs millimètres. On l'observe habituellement en cristaux prismatiques incolores terminés par des faces du rhomboctaèdre $\{111\}$, ce qui confère à ces cristaux une morphologie lancéolée caractéristique (Figures 4A et 4B). Plus rarement, on peut observer des cristaux prismatiques trapus blanc laiteux (Figure 4D) ou des cristaux losangiques tabulaires (Figure 4C). Considérée comme rare en Belgique (Hatert et al., 2002), l'anglésite a cependant été reconnue dans plusieurs autres gisements ardennais : La Roche (Blondieau \& Hatert, 2009), Bure (Blondieau, 2011) et Chanly. 
Bulletin de la Société Royale des Sciences de Liège, Vol. 86, articles, 2017, p. 169 - 201
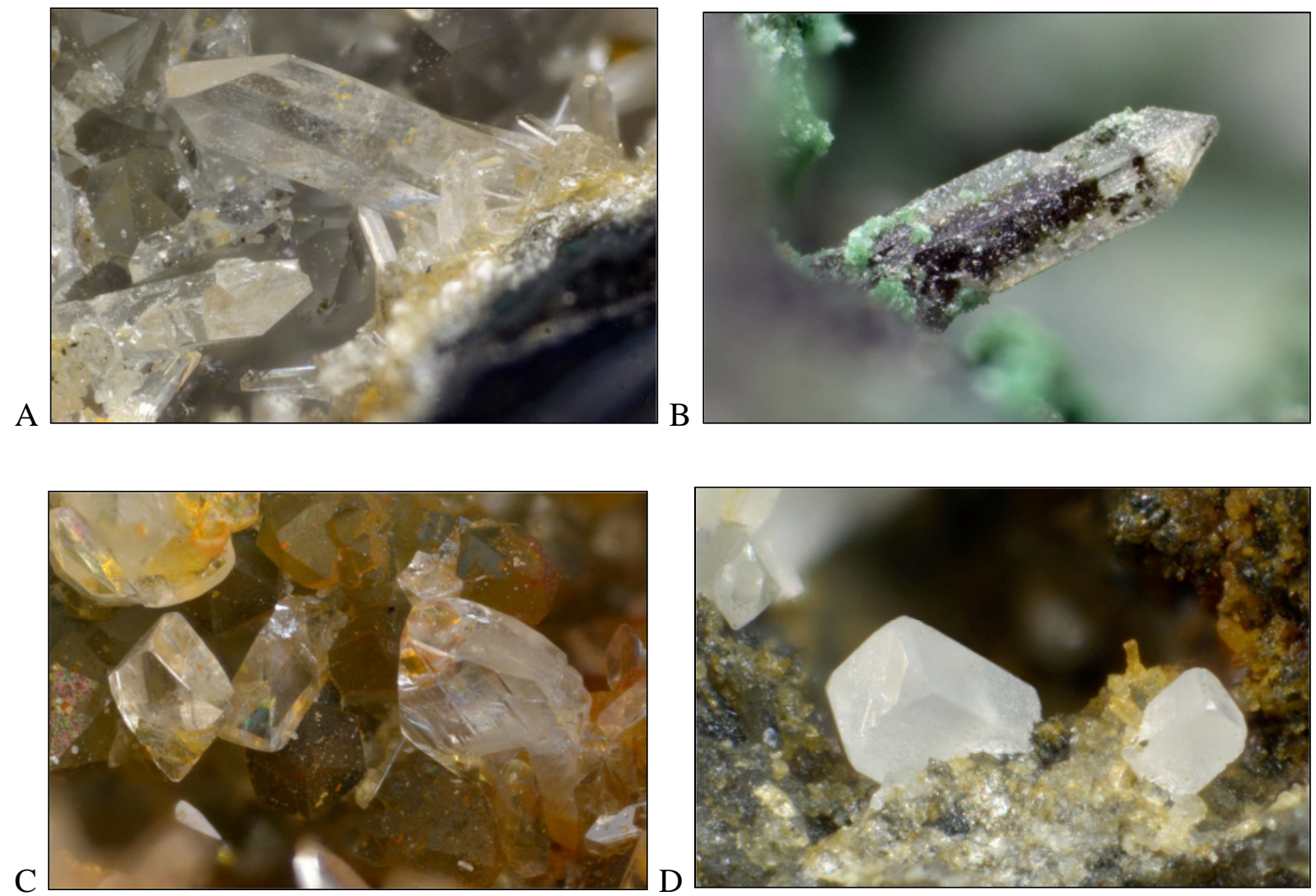

Fig. 4 : Anglésite de Masbourg. (A) et (B) Cristaux lancéolés incolores. (C) Cristaux incolores. (D) Cristaux prismatiques laiteux. $\mathrm{L}=1,6 \mathrm{~mm}(\mathrm{~A}) ; 1,05 \mathrm{~mm}(\mathrm{~B}) ; 1,5 \mathrm{~mm}(\mathrm{C}) ; 0,8 \mathrm{~mm}(\mathrm{D})$. (Photos P. Chollet).

\subsection{Aragonite, $\mathrm{CaCO}_{3}$}

L'aragonite est un membre rare de la paragenèse de Masbourg. Elle se présente en petite boules blanches montrant, sur cassure, une structure fibroradiée (Figure 5). Le minéral est identifié par diffraction des rayons $\mathrm{X}$. 

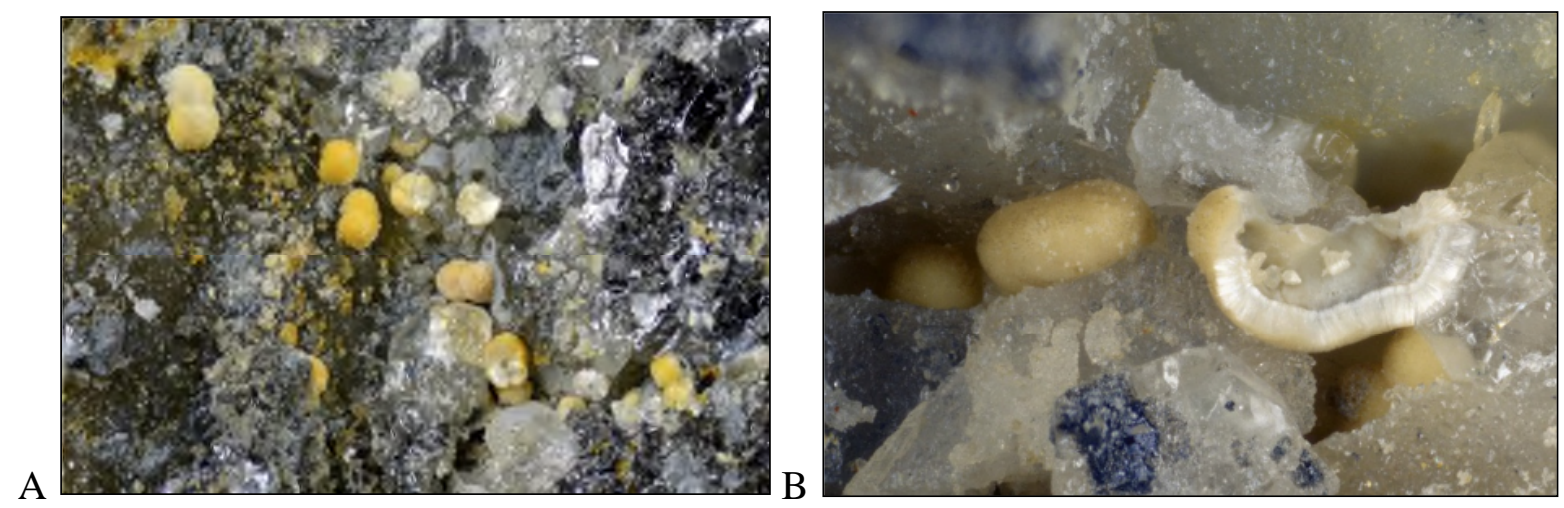

Fig. 5 : Aragonite de Masbourg. L = 3,6 mm (A) ; 1,6 mm (B). (Photos P. Chollet).

\subsection{Aurichalcite, $(\mathrm{Zn}, \mathrm{Cu})_{5}\left(\mathrm{CO}_{3}\right)_{2}(\mathrm{OH})_{6}$}

Sur un seul échantillon, des petites boules bleu pâle ont visuellement été identifiées comme de l'aurichalcite. Une analyse par diffraction des rayons $\mathrm{X}$, ainsi que des analyses chimiques qualitatives par EDS ayant mis en évidence les éléments $\mathrm{Zn}, \mathrm{Cu}, \mathrm{C}$ et $\mathrm{O}$, confirment l'espèce. En microscopie électronique à balayage, la surface des boules montre un empilement serré de minuscules cristaux lamellaires (Figure 6).
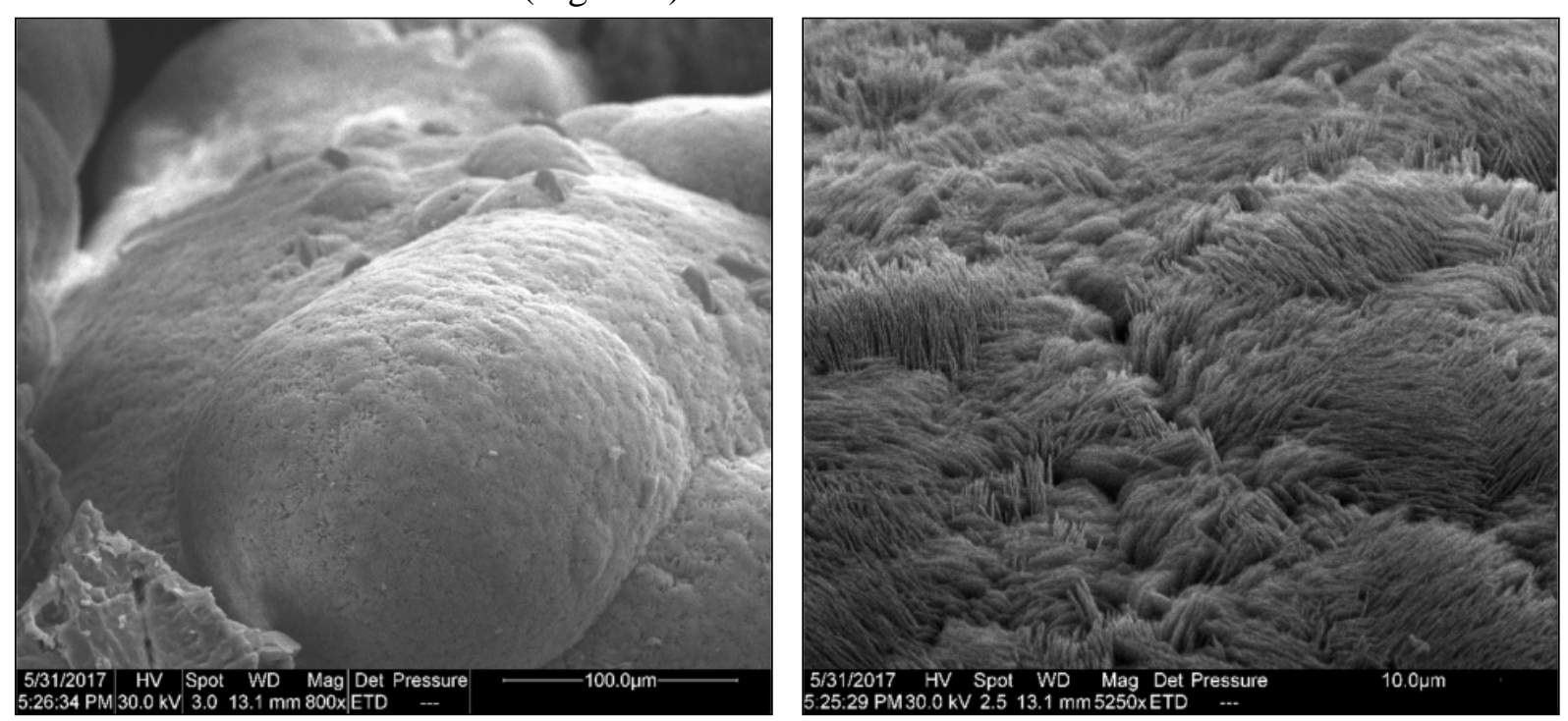

Fig. 6 : Aurichalcite de Masbourg. Échantillon MAS-01, photographie au microscope électronique à balayage, mode électrons secondaires.

\subsection{Brochantite, $\mathrm{Cu}_{4}\left(\mathrm{SO}_{4}\right)(\mathrm{OH})_{6}$}

La brochantite forme des petites plages vertes dans lesquelles on peut observer de minuscules cristaux assez mal formés (Figures 7A, 7B). Elle est fréquemment associée à la linarite. Le minéral est identifié par diffraction des rayons $\mathrm{X}$, ainsi que par des analyses chimiques qualitatives EDS qui mettent en évidence les éléments majoritaires $\mathrm{Cu}, \mathrm{S}$ et $\mathrm{O}$, confirmant ainsi 
Bulletin de la Société Royale des Sciences de Liège, Vol. 86, articles, 2017, p. 169 - 201

l'espèce. En microscopie électronique à balayage, on observe des cristaux fort aplatis, voire lamellaires (Figures 7C, 7D).

A
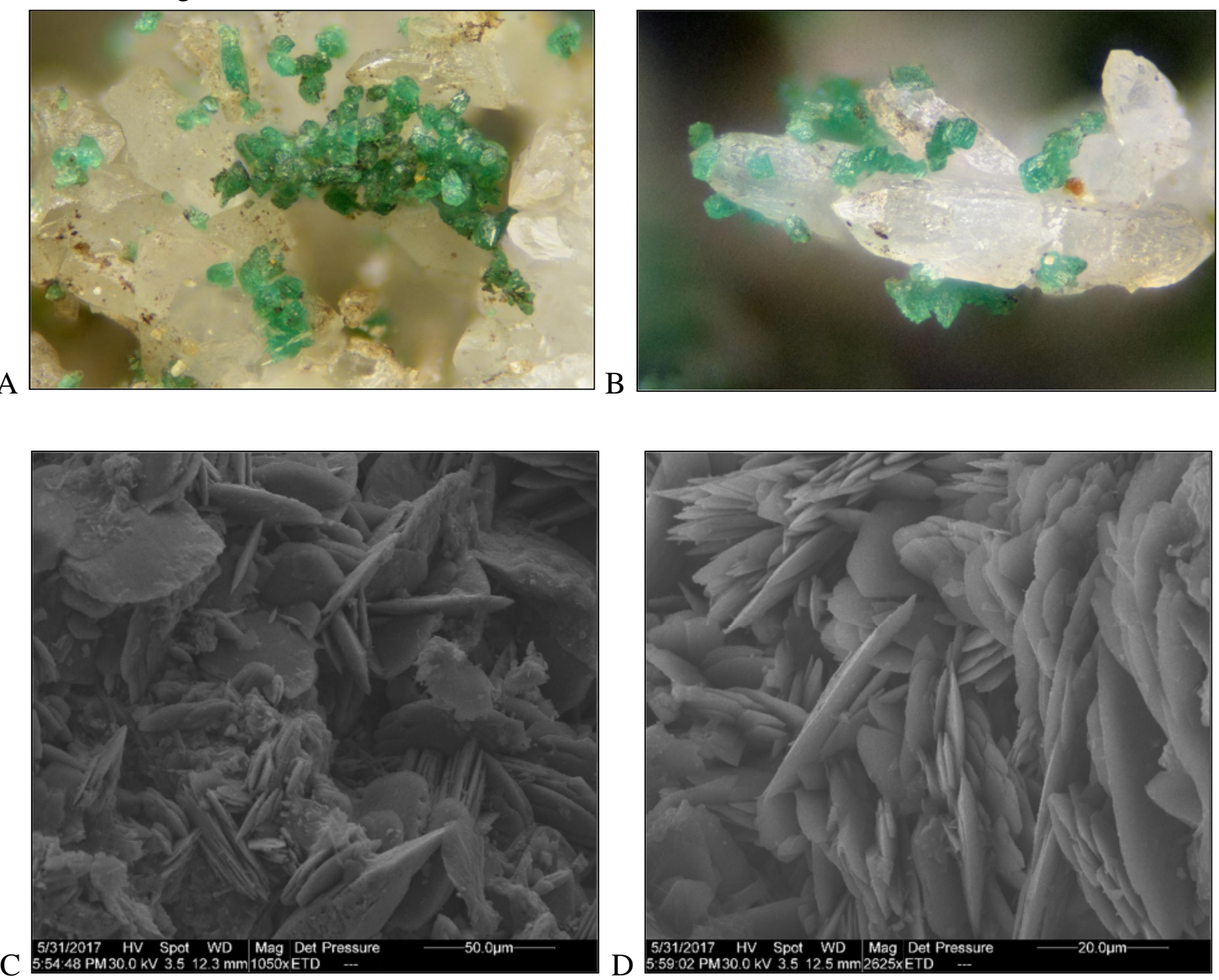

Fig 7. : Brochantite de Masbourg. (A) et (B) Petits cristaux sur quartz laiteux. $L=0,84 \mathrm{~mm}$ (A) et $0,72 \mathrm{~mm}$ (B). (Photos P. Chollet). (C) et (D) Échantillon MAS 05. Photographies au microscope électronique à balayage, mode électrons secondaires.

\subsection{Calcite, $\mathrm{CaCO}_{3}$}

Une veinule blanche, traversant un échantillon riche en galène, a été reconnue par diffraction des rayons $\mathrm{X}$ comme constituée de calcite, mais aucune forme cristalline n'a pu être observée. Le minéral n'a été observé que sur quelques échantillons.

\subsection{Calédonite, $\mathrm{Cu}_{2} \mathrm{~Pb}_{5}\left(\mathrm{SO}_{4}\right)_{3}\left(\mathrm{CO}_{3}\right)(\mathrm{OH})_{6}$}

La calédonite est observée en petites boules ou en petits bouquets de cristaux bleu-ciel, assez pâles et très minces (Figures $8 \mathrm{~A}$ et $8 \mathrm{~B}$ ). Le minéral est peu fréquent à Masbourg, et les plages observées ne dépassent guère quelques millimètres de longueur. Elle s'observe dans des parties corrodées de la galène, en association avec la linarite et la cérusite. Le minéral est identifié par 
Bulletin de la Société Royale des Sciences de Liège, Vol. 86, articles, 2017, p. 169 - 201

diffraction des rayons $\mathrm{X}$, et également grâce aux analyses chimiques qualitatives qui mettent en évidence les éléments majoritaires $\mathrm{Pb}, \mathrm{Cu}, \mathrm{C}, \mathrm{S}$ et $\mathrm{O}$, ainsi que $\mathrm{Sr}$ et $\mathrm{Al}$ fort minoritaires. $\mathrm{La}$ calédonite est une espèce nouvelle pour la Belgique. En microscopie électronique à balayage, on peut observer des agrégats sphériques constitués par l'association de nombreux cristaux lamellaires (Figure 8C) montrant, à fort grossissement, une surface assez corrodée (Figures 8D et $8 \mathrm{E})$.

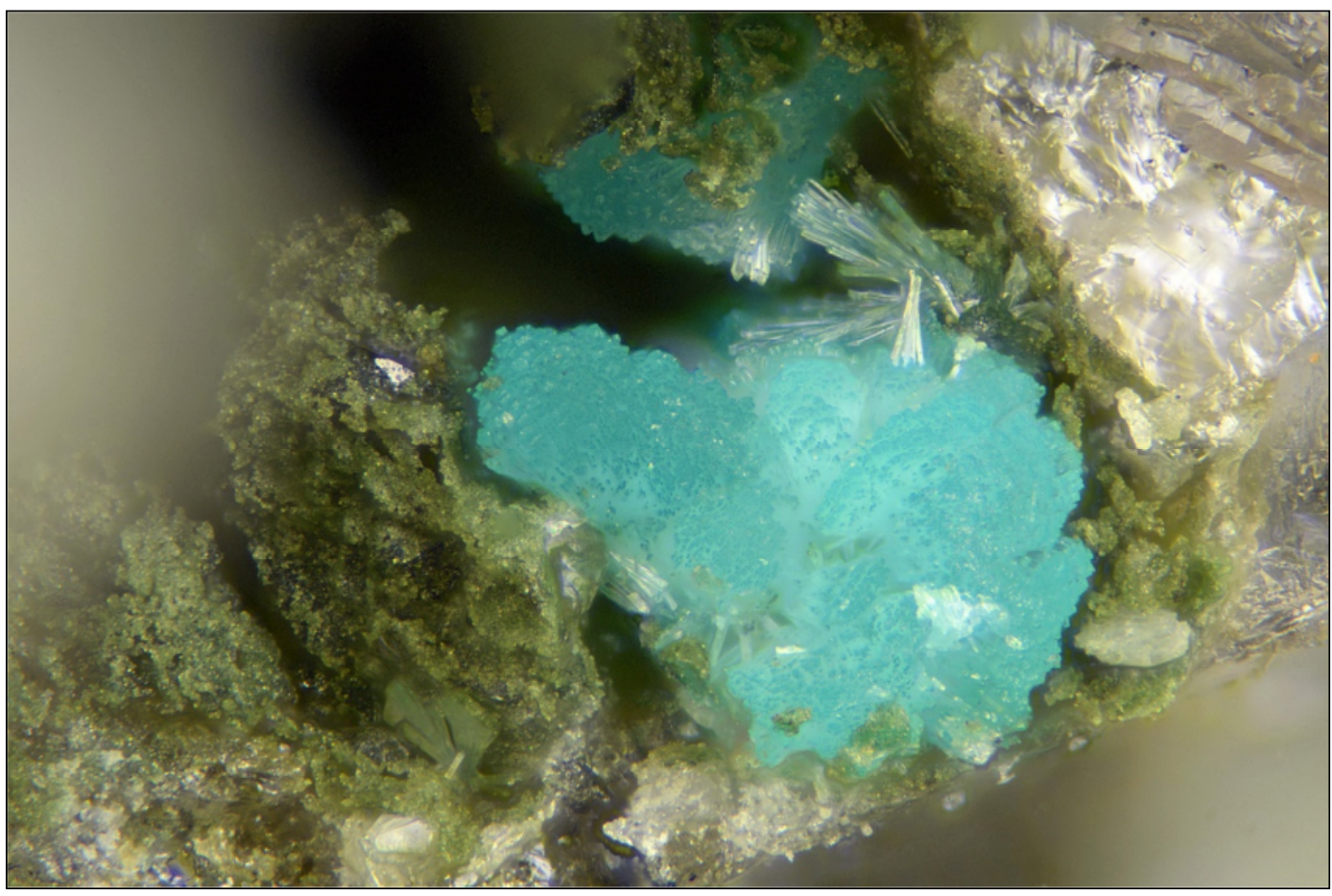


Bulletin de la Société Royale des Sciences de Liège, Vol. 86, articles, 2017, p. 169 - 201
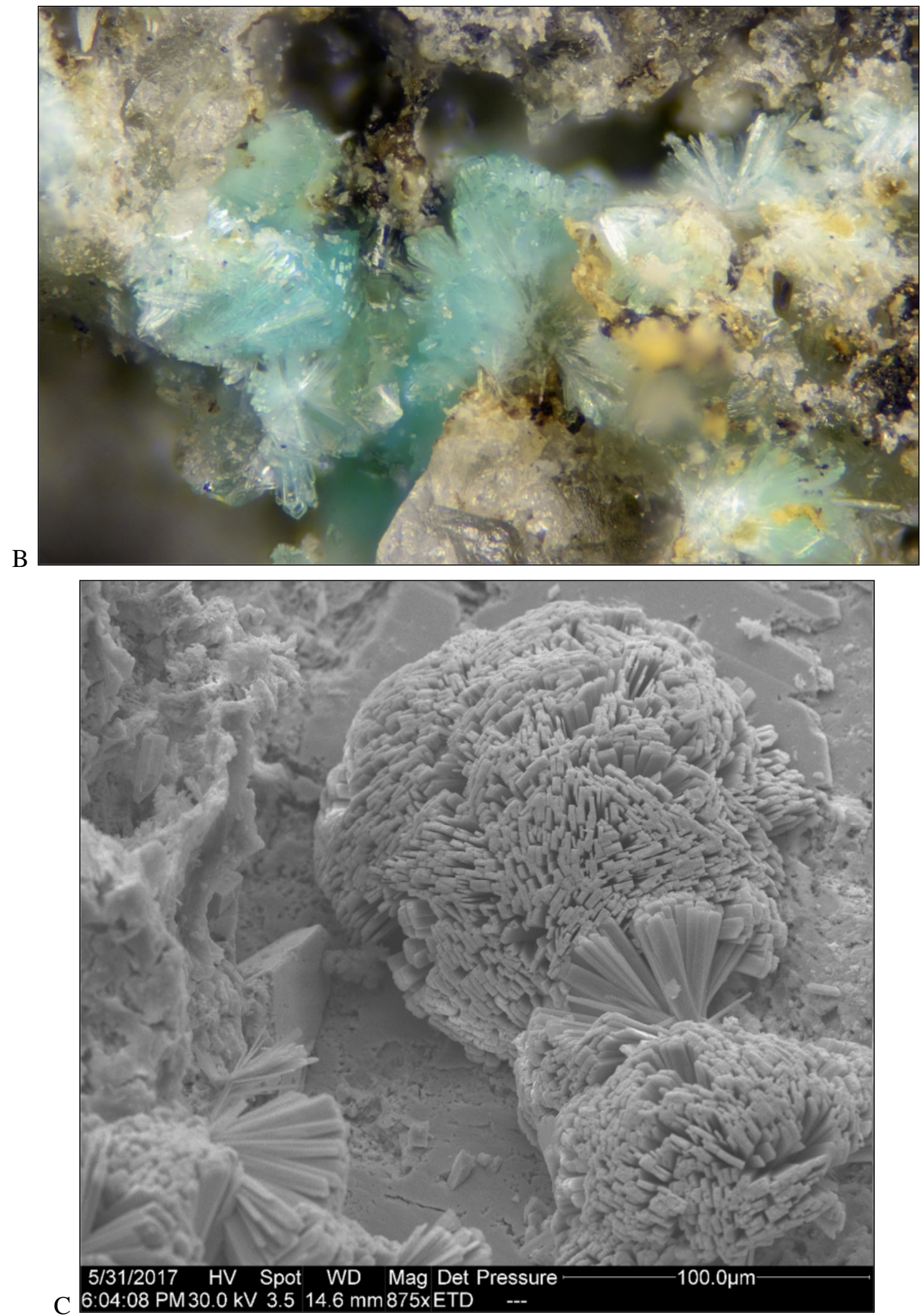

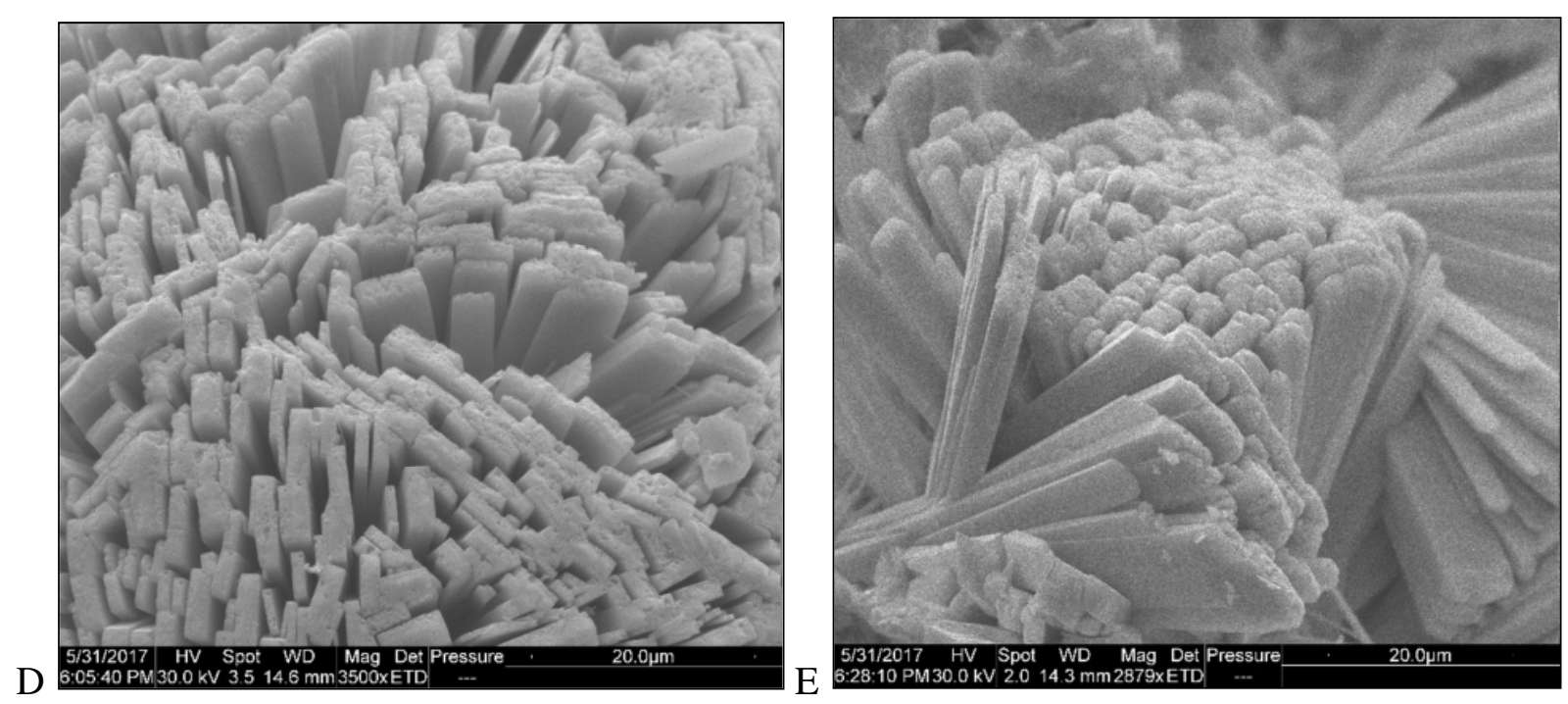

Fig. 8 : Calédonite de Masbourg. (A) Agrégat constitué de nombreux cristaux lamellaires. L = 0,87 mm. (B) Cristaux en gerbes. L=0,79 mm. (A) et (B), (Photos P. Chollet). (C), (D), (E) Échantillon MAS 06. Photographies au microscope électronique à balayage, mode électrons secondaires.

\subsection{Cérusite, $\mathrm{PbCO}_{3}$}

La cérusite est un minéral d'altération fréquent de la galène. À Masbourg, on l'observe fréquemment en enduits blancs sur galène, ou en injections vitreuses incolores ou blanc laiteux dans les roches encaissantes. Parfois aussi, on la rencontre en plages vitreuses de couleur noire, dans la galène. Cette couleur sombre est sans doute due à la présence de minuscules inclusions de galène dans la cérusite. Le minéral ne s'exprime que rarement en beaux cristaux à Masbourg. La Figure 9B montre un cristal maclé sur du quartz, avec de la galène corrodée. La cérusite est identifiée par diffraction des rayons X ; on la voit parfois associée à la calédonite (Figure 9A). 
Bulletin de la Société Royale des Sciences de Liège, Vol. 86, articles, 2017, p. 169 - 201
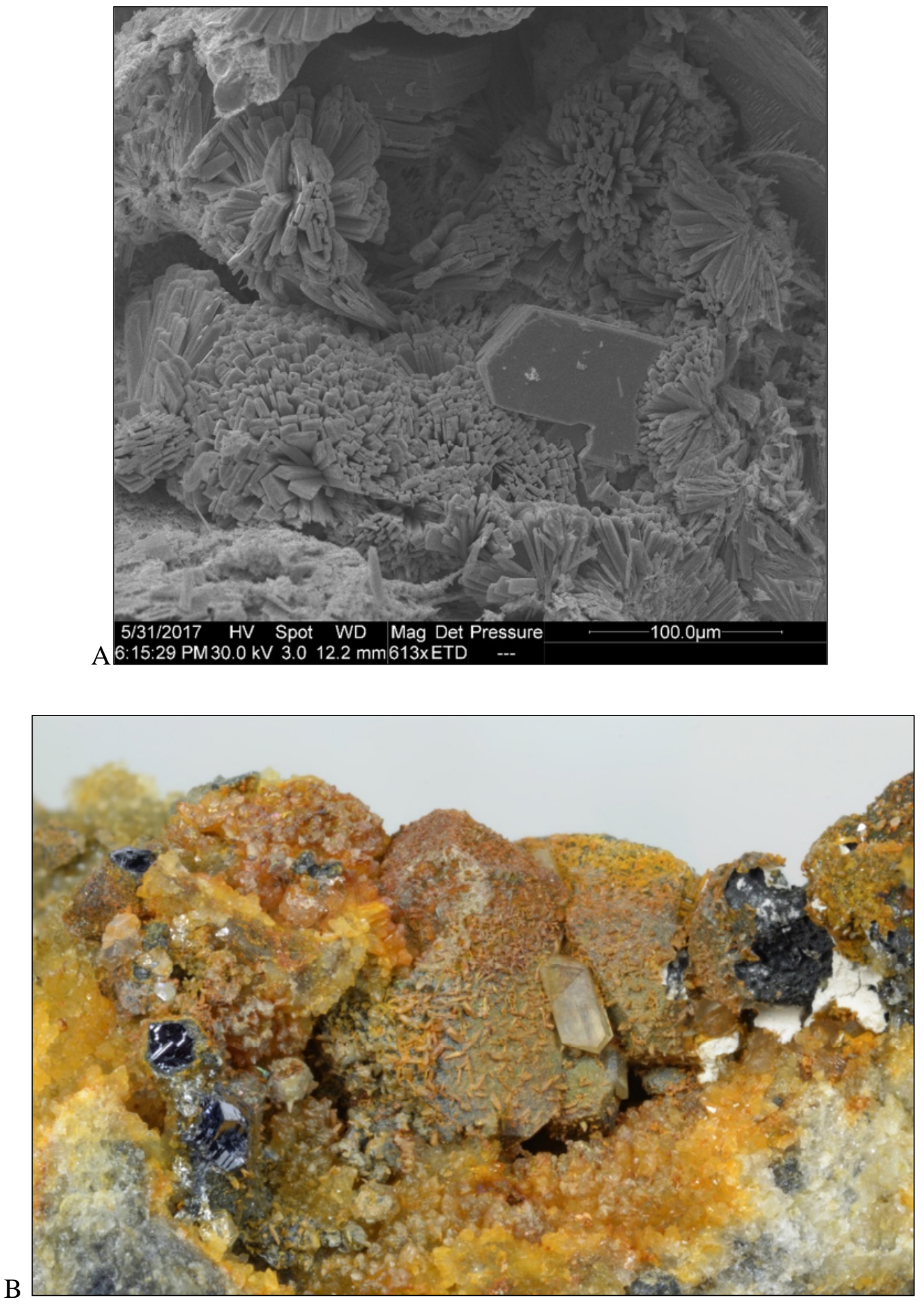

Fig. 9 : Cérusite de Masbourg. (A) Cristal de cérusite sur calédonite. Échantillon MAS-06c. Photographie an microscope électronique à balayage, mode électrons secondaires. (B) Cristal maclé sur galène. $\mathrm{L}=5,7 \mathrm{~mm}$. Photo $\mathrm{P}$. Chollet. 
Bulletin de la Société Royale des Sciences de Liège, Vol. 86, articles, 2017, p. 169 - 201

\subsection{Chalcopyrite, $\mathrm{CuFeS}_{2}$}

Certains échantillons très quartzeux montrent parfois de belles plages de chalcopyrite en association avec la galène (Figure 10). La chalcopyrite, identifiée visuellement, est confirmée par diffraction des rayons $\mathrm{X}$. Dans la région, la chalcopyrite n'est pas rare dans les petits filons à gangue quartzeuse. C'est notamment le cas à Chanly, Tellin et Ambly.

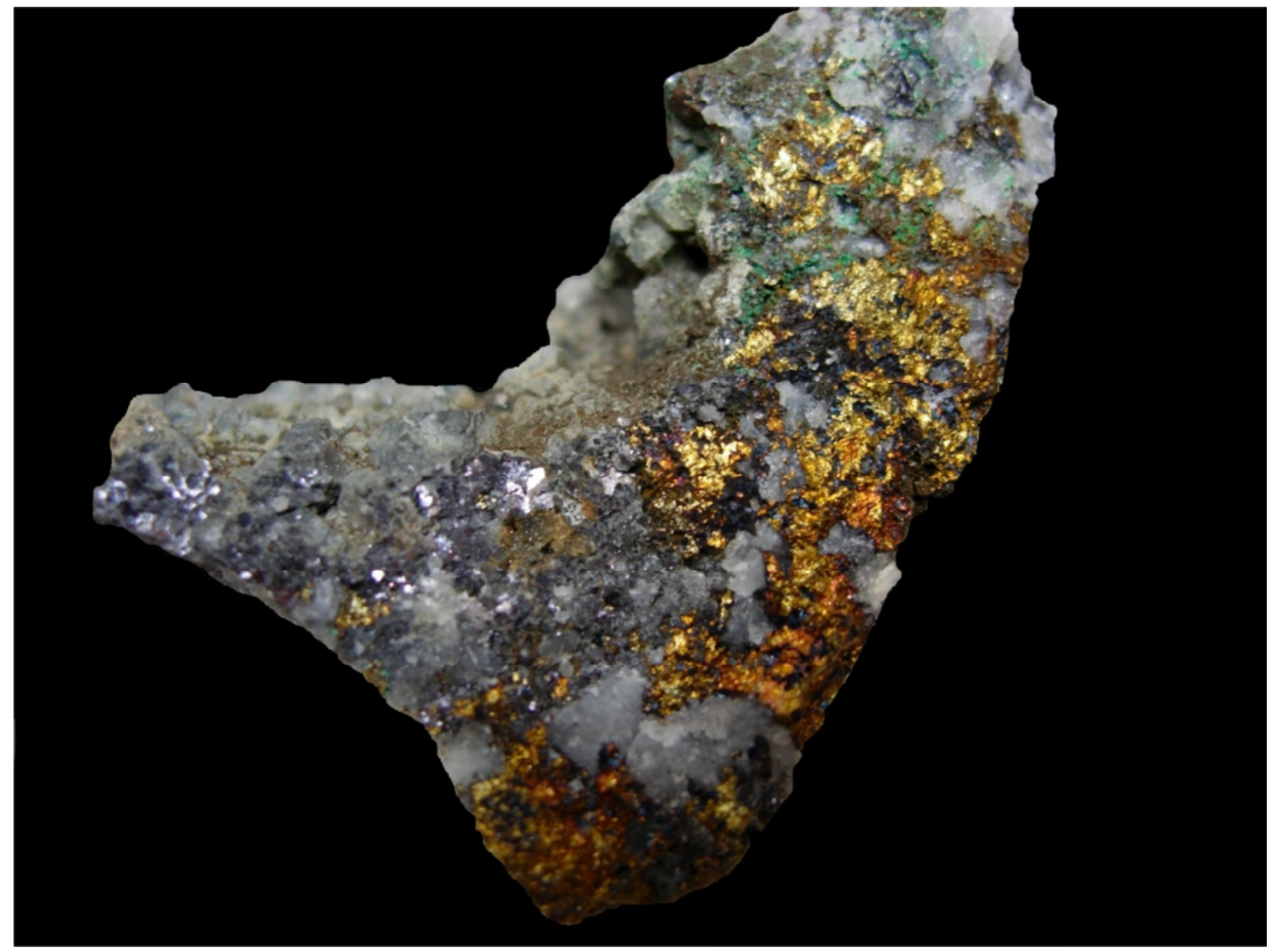

Fig. 10 : Association de galène et de chalcopyrite dans du quartz laiteux. Masbourg, $\mathrm{L}=3 \mathrm{~cm}$.

\subsection{Chamosite, $\left(\mathrm{Fe}^{2+}, \mathrm{Mg}, \mathrm{Al}, \mathrm{Fe}^{3+}\right)_{6}(\mathrm{Si}, \mathrm{Al})_{4} \mathrm{O}_{10}(\mathrm{OH}, \mathrm{O})_{8}$}

Sur des échantillons de roche recueillis dans les haldes de Masbourg, on peut observer des boules vert foncé visuellement identifiées comme une chlorite, ce que confirment les analyses par diffraction des rayons X. Les analyses chimiques qualitatives par EDS indiquent la présence des éléments majoritaires $\mathrm{Si}, \mathrm{Al}, \mathrm{Fe}$ et $\mathrm{Mg}$. Bien qu'étant non quantitative, l'analyse met en évidence une présence de fer très nettement supérieure à celle de magnésium, ce qui permet d'attribuer cette chlorite à une chamosite et non à un clinochlore (Figures $11 \mathrm{~A}$ et 11B). 
Bulletin de la Société Royale des Sciences de Liège, Vol. 86, articles, 2017, p. 169 - 201
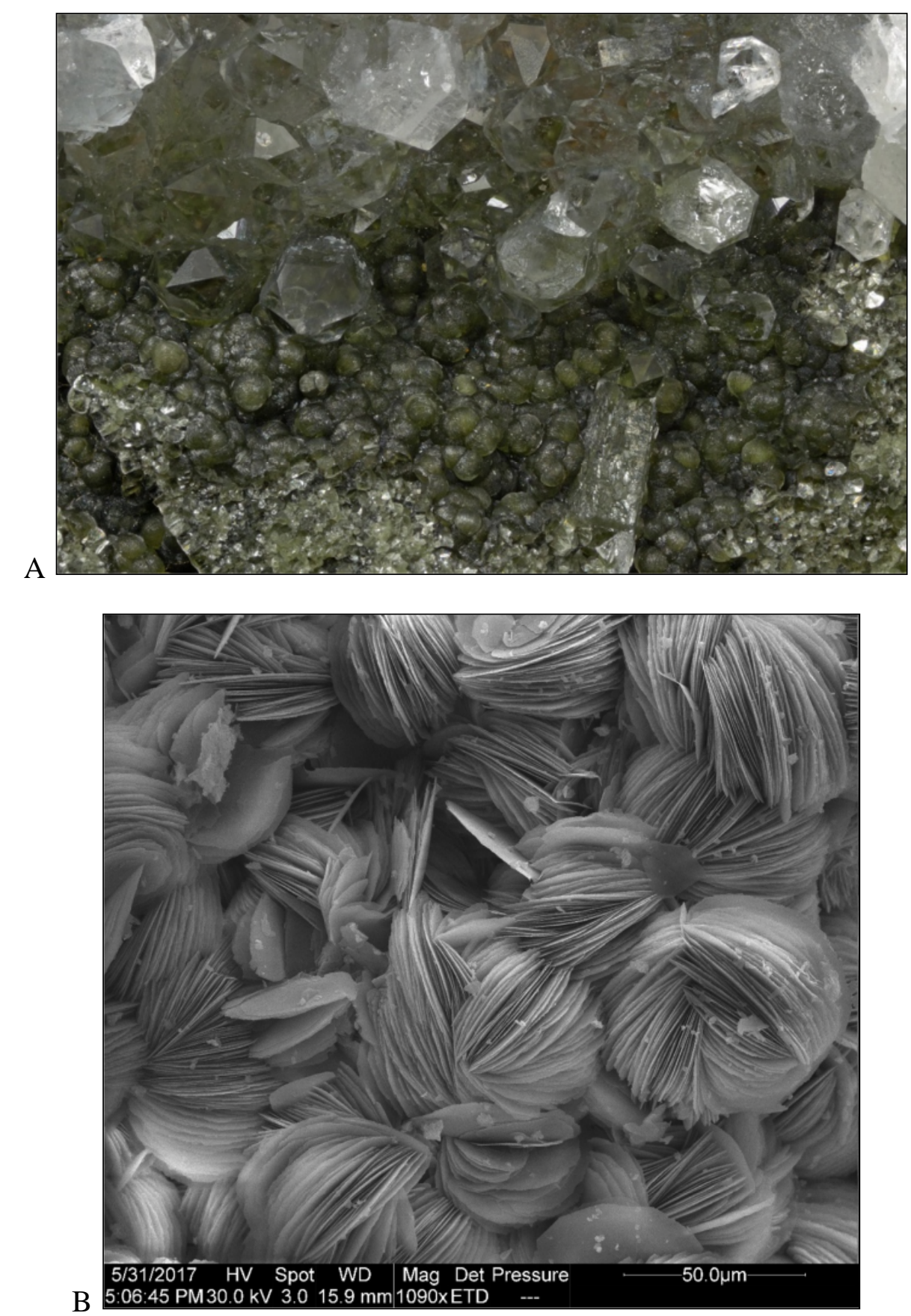

Fig. 11 : Chamosite de Masbourg. (A) Agrégats de chamosite et quartz de Masbourg. L = 7,0 mm. (Photo P. Chollet). (B) Échantillon MAS-03. Photographie au microscope électronique à balayage, mode électrons secondaires. 
Bulletin de la Société Royale des Sciences de Liège, Vol. 86, articles, 2017, p. 169 - 201

\subsection{Covellite, $\mathrm{CuS}$}

La covellite, identifiée par diffraction des rayons $X$, se présente en efflorescences bleu métallique avec reflets violacés, autour de la chalcopyrite et de la galène. Il s'agit d'une espèce de postformation, qui apparaît sur les échantillons mis en collection.

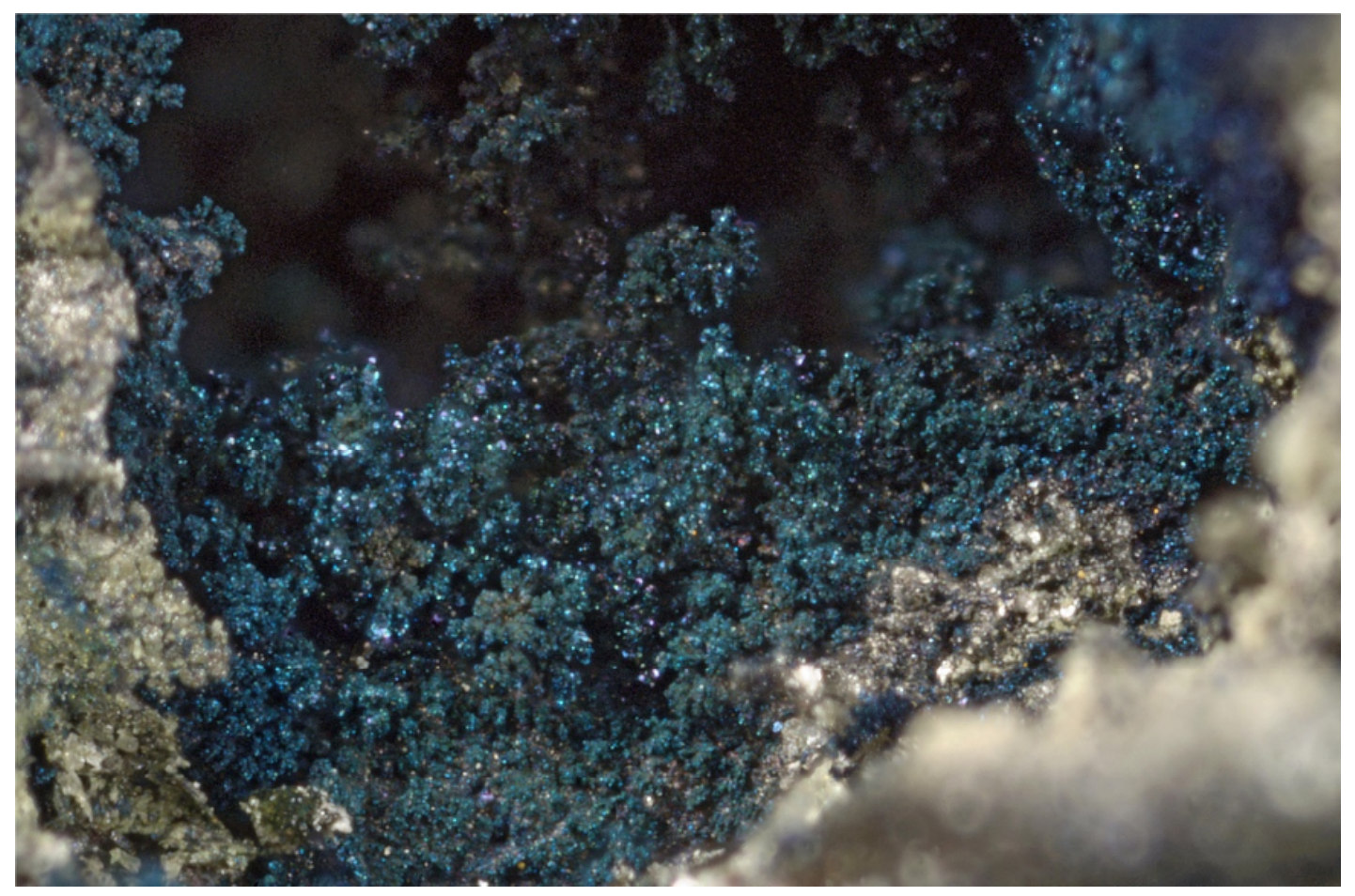

Fig. 12 : Covellite de Masbourg. $\mathrm{L}=1,85 \mathrm{~mm}$. (Photo P. Chollet).

\subsection{Cuprite, $\mathrm{Cu}_{2} \mathrm{O}$}

La cuprite est un membre très rare de la paragenèse de Masbourg. Le minéral est identifié visuellement sur un unique échantillon (Figure 13).

\subsection{Dickite, $\mathrm{Al}_{2} \mathrm{Si}_{2} \mathrm{O}_{5}(\mathrm{OH})_{4}$}

Des fractures de la roche altérée sont remplies d'un minéral blanc de texture argileuse. L'analyse par diffraction des rayons $\mathrm{X}$ indique qu'il s'agit de dickite. Dans la région, ce minéral d'altération a également été observé à Chanly. A Masbourg, le minéral se montre parfois aussi en amas de minuscules cristaux incolores très minces, de forme hexagonale. 


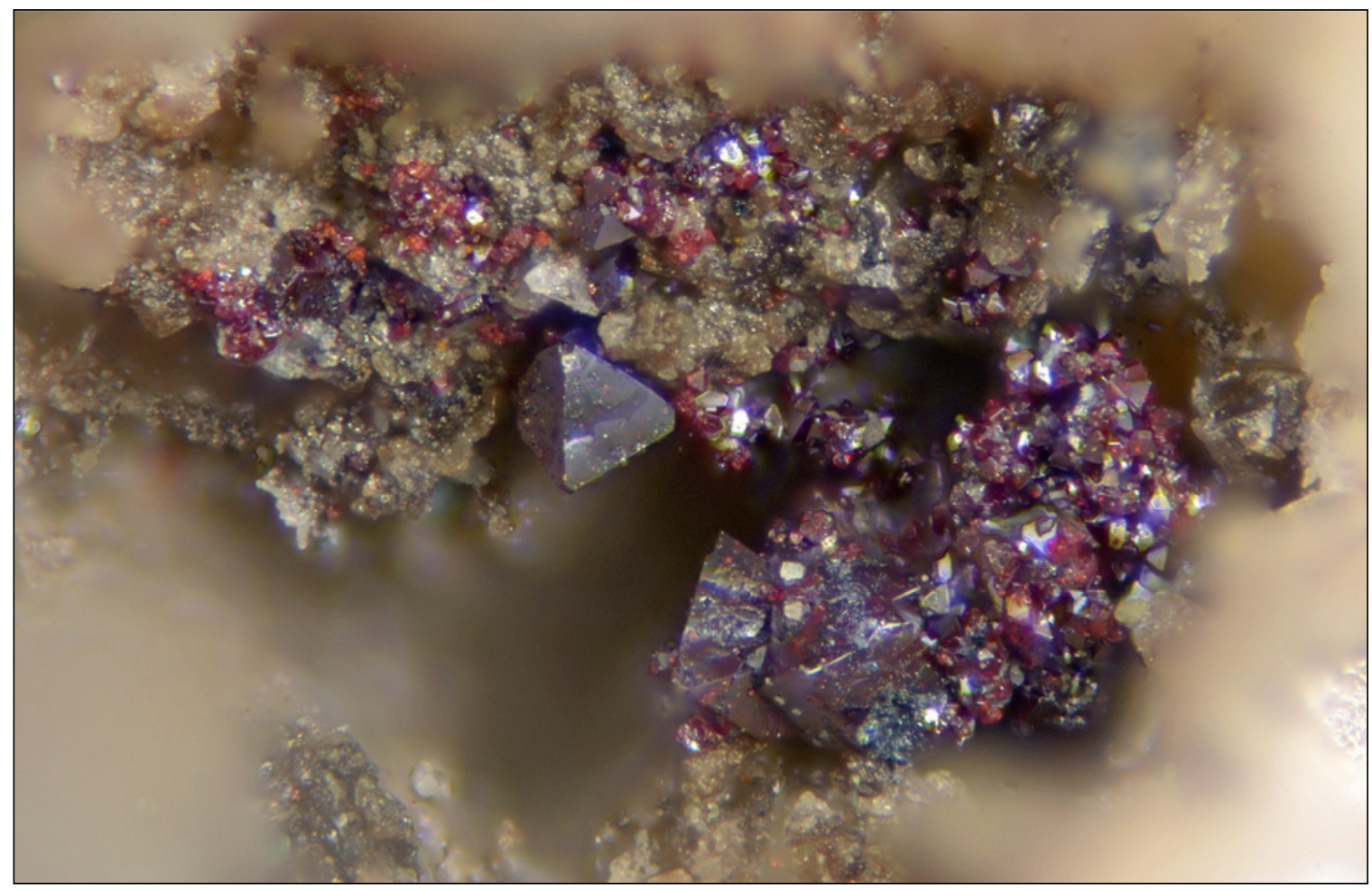

Fig. 13 : Cuprite de Masbourg. $\mathrm{L}=0,65 \mathrm{~mm}$. (Photo P. Chollet).

\subsection{Dolomite, $\mathrm{CaMg}\left(\mathrm{CO}_{3}\right)_{2}$}

Un minéral du groupe de la dolomite est identifié par diffraction des rayons $\mathrm{X}$. Ce carbonate fait fréquemment partie de la paragenèse des filons métalliques mais, en Ardenne, il est souvent fortement altéré dans les zones proches de la surface. Nous avons cependant observé un échantillon présentant, sur cassure, des plages blanc-rosée parsemées de quelques cristaux de forme rhomboédrique caractéristique. Le plus souvent, ces cristaux ne sont observés qu'à l'état de "reliques" brun rouille, une teinte qui trahit la présence de fer dans cette dolomite, ce qui est d'ailleurs bien souvent le cas en Ardenne. La Figure 14 montre des pseudomorphoses très fragiles de cristaux d'habitus rhomboédrique, qui étaient vraisemblablement de la dolomite. La todorokite est souvent associée à ces pseudomorphoses. 


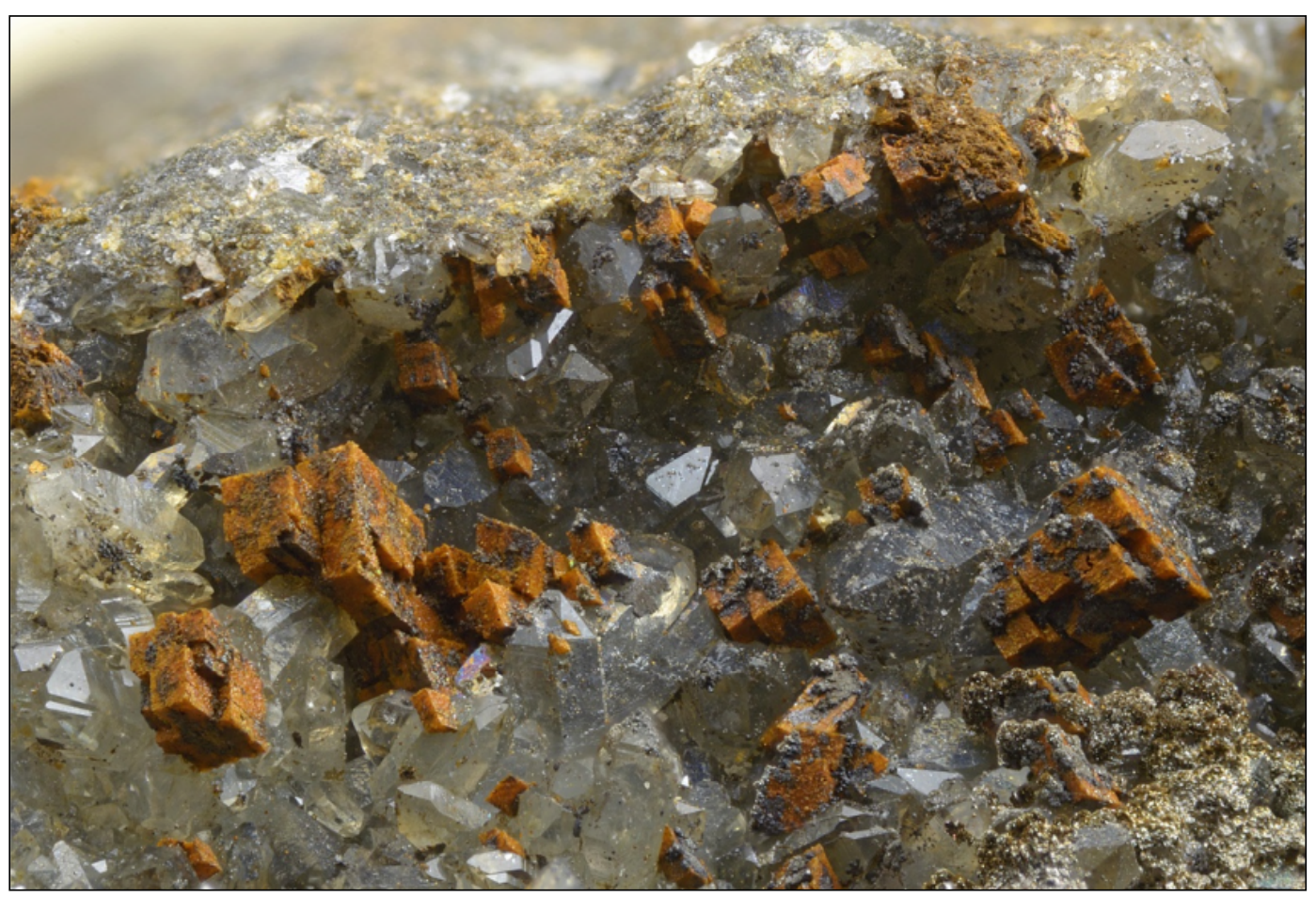

Fig. 14 : Pseudomorphoses brun-rouille de cristaux rhomboédriques sur quartz, Masbourg. $\mathrm{L}=5,1 \mathrm{~mm}$. (Photo P. Chollet).

\subsection{Galène, $\mathrm{PbS}$}

Lors de nos recherches, nous avons pu retrouver en relative abondance des morceaux de galène, dont certains de belle taille. Ces fragments atteignent $10 \mathrm{~cm}$ de longueur, pour une épaisseur de 3 $\mathrm{cm}$; il s'agit sans doute de la puissance d'un des filons exploités à l'époque. Des cristaux corrodés de plusieurs centimètres d'arête ont également été retrouvés (Figure 15). Ils montrent la forme du cube affecté par les faces $\{111\}$ de l'octaèdre.

Dans le quartz blanc, on observe également des plages de galène souvent associée à la chalcopyrite. Enfin, il est parfois possible d'observer aussi de minuscules inclusions arrondies formant des gouttelettes grises métalliques, dans des petits cristaux de quartz. Il s'agit sans doute aussi de galène. 
Bulletin de la Société Royale des Sciences de Liège, Vol. 86, articles, 2017, p. 169 - 201

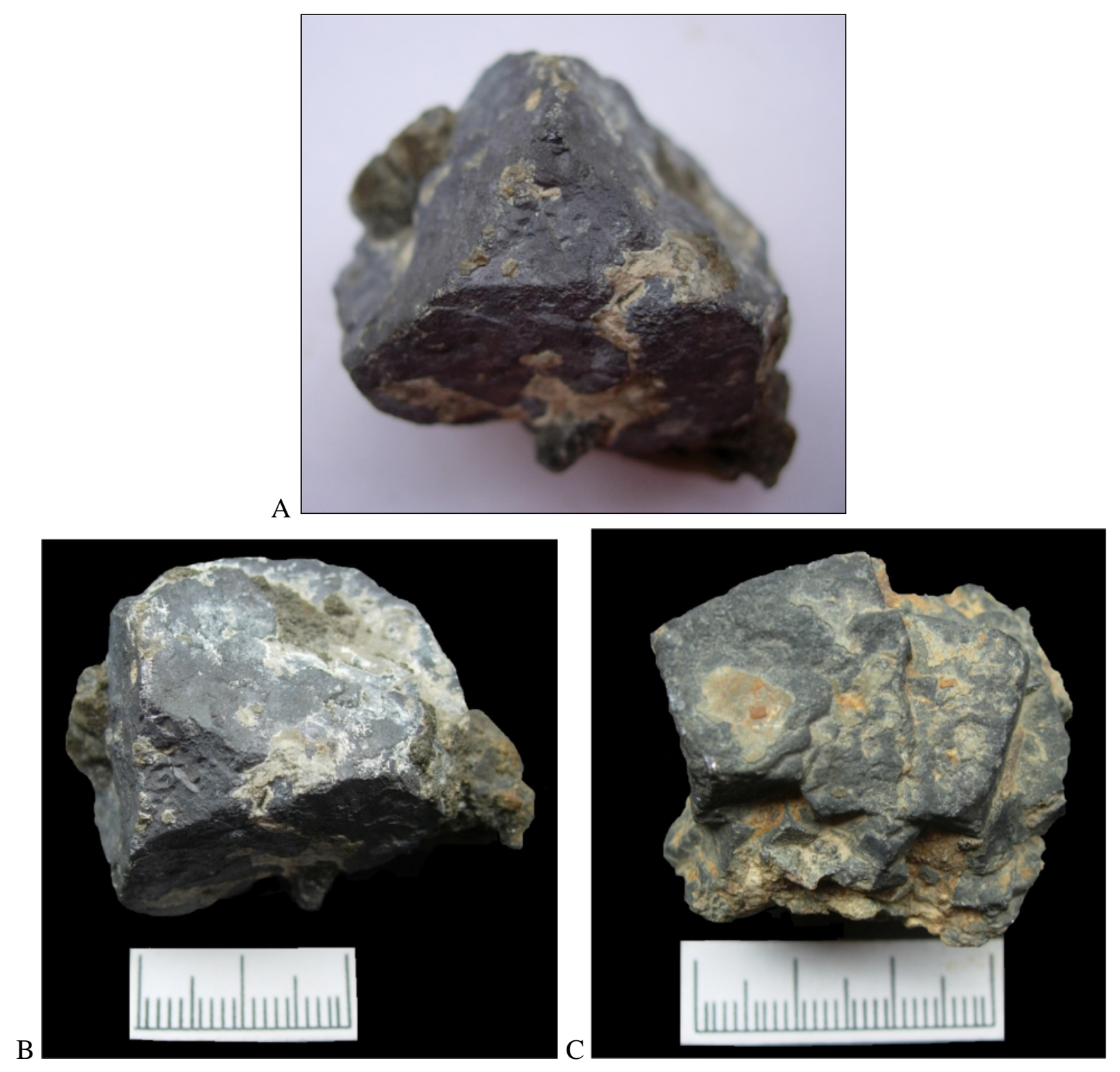

Fig 15 : Gros cristaux de galène corrodée, Masbourg. (A) $\mathrm{L}=3 \mathrm{~cm}$.

\subsection{Hydrozincite, $\mathrm{Zn}_{5}\left(\mathrm{CO}_{3}\right)_{2}(\mathrm{OH})_{6}$}

Sur une grande plage de sphalérite brune associée à de la galène, on peut observer une croûte blanche d'épaisseur millimétrique qui, sur cassure, montre une structure fibreuse. Localement, ce minéral se présente en petites sphérules. Visuellement déterminé comme hydrozincite, l'identification est confirmée par diffraction des rayons X. Ce minéral n'a été observé que sur un seul échantillon. 
Bulletin de la Société Royale des Sciences de Liège, Vol. 86, articles, 2017, p. 169 - 201

\subsection{Linarite, $\mathrm{CuPb}\left(\mathrm{SO}_{4}\right)(\mathrm{OH})_{2}$}

La linarite est observée à Masbourg en petits cristaux bleus (Figure 16) et est identifiée par diffraction des rayons $\mathrm{X}$, ainsi que par des analyses chimiques qualitatives qui mettent en évidence la présence des éléments majoritaires $\mathrm{Pb}, \mathrm{Cu}, \mathrm{S}$ et $\mathrm{O}$. On l'observe souvent en association avec la cérusite, la brochantite et, plus rarement, avec la calédonite. La linarite n'est pas une espèce fréquente en Belgique, mais elle a déjà été rencontrée à Bastogne (Hatert, 2002), à La Roche-en-Ardenne (Blondieau \& Hatert, 2009), dans le filon de Bleiberg à Schimper (Blondieau \& Polrot, 2011) et à Chanly.

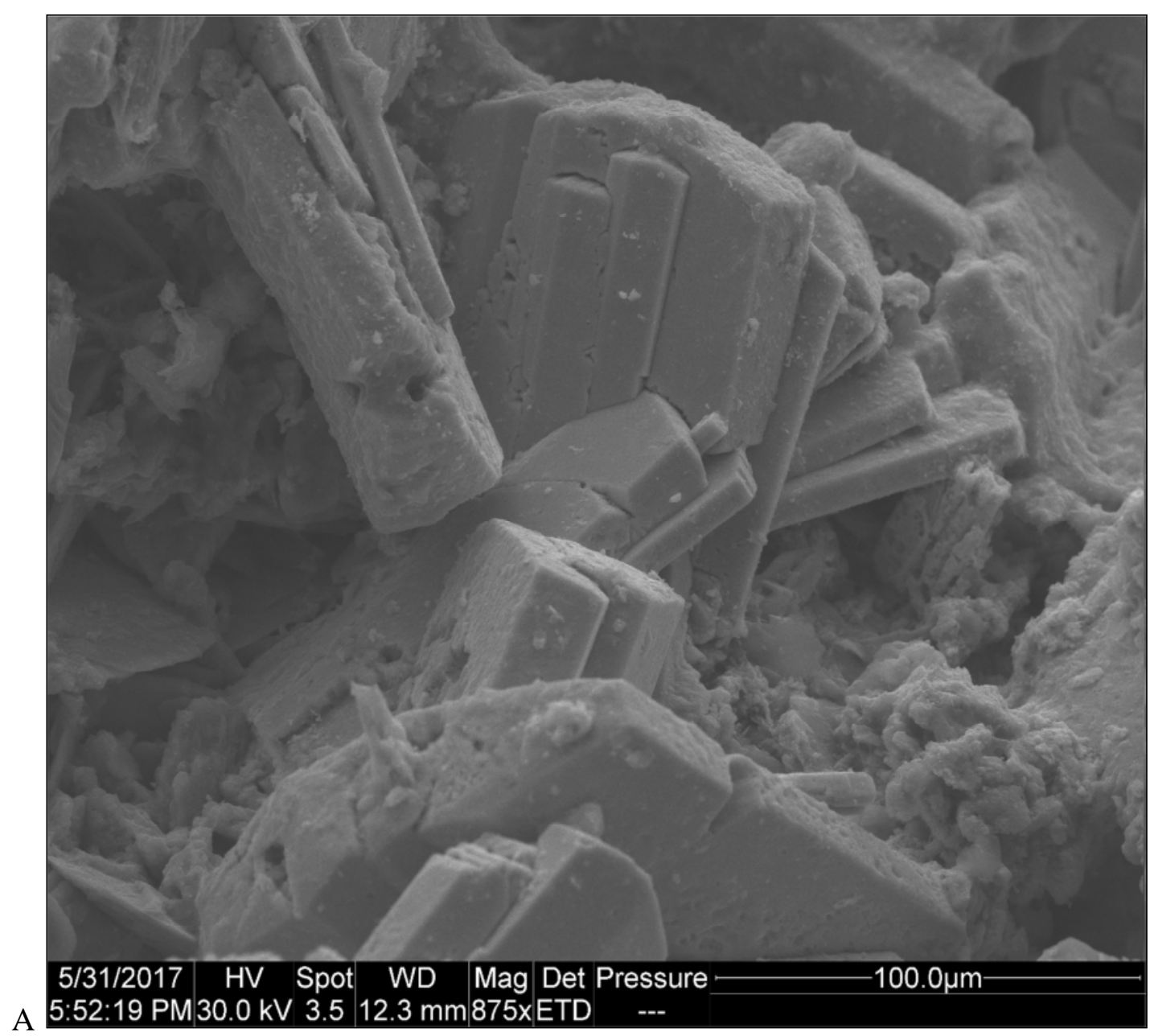


Bulletin de la Société Royale des Sciences de Liège, Vol. 86, articles, 2017, p. 169 - 201
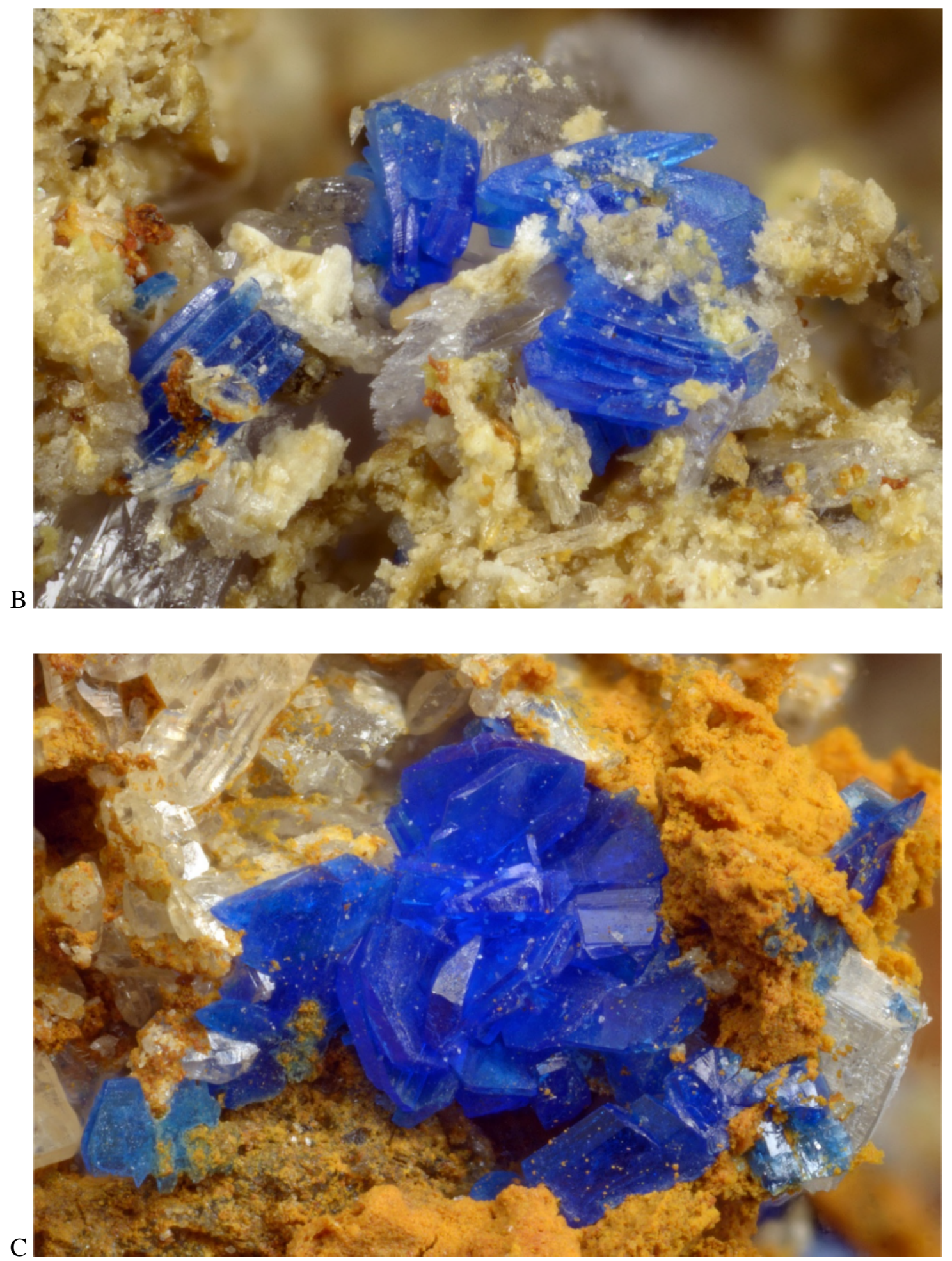

Fig 16 : Linarite de Masbourg. (A) Échantillon MAS 05c. Photographie au microscope électronique à balayage, mode électrons secondaires. (B) $\mathrm{L}=1,45 \mathrm{~mm}$. (C) $\mathrm{L}=2,1 \mathrm{~mm}$. (B) et (C) (Photos P. Chollet). 
Bulletin de la Société Royale des Sciences de Liège, Vol. 86, articles, 2017, p. 169 - 201

\subsection{Malachite, $\mathrm{Cu}_{2}\left(\mathrm{CO}_{3}\right)\left(\mathrm{OH}_{2}\right.$}

La malachite se forme à proximité de la chalcopyrite dont elle dérive, et s'observe en enduits ou en petits globules verts (Figure 17). Parfois aussi, elle cristallise en efflorescences dans des cavités de la galène (Figure 18). L'identification du minéral est effectuée visuellement et est confirmée par diffraction des rayons $\mathrm{X}$.

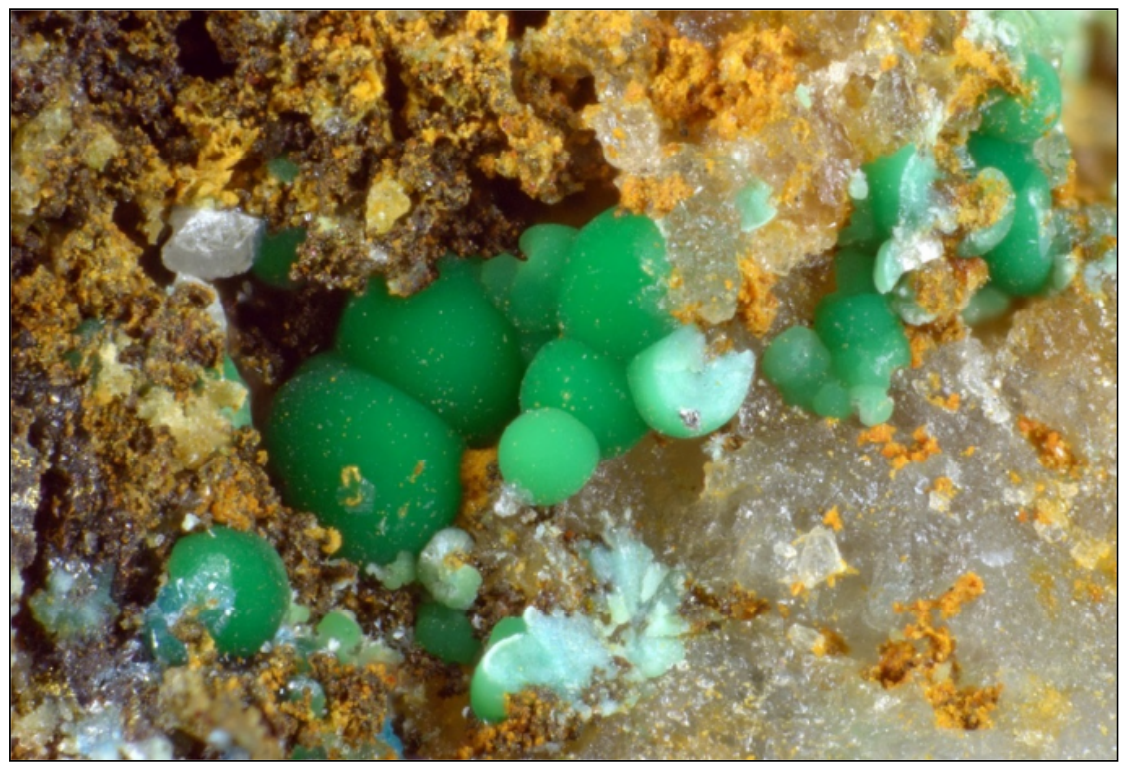

Fig. 17 : Malachite en petits globules, Masbourg. $\mathrm{L}=1,45 \mathrm{~mm}$. (Photo P. Chollet).

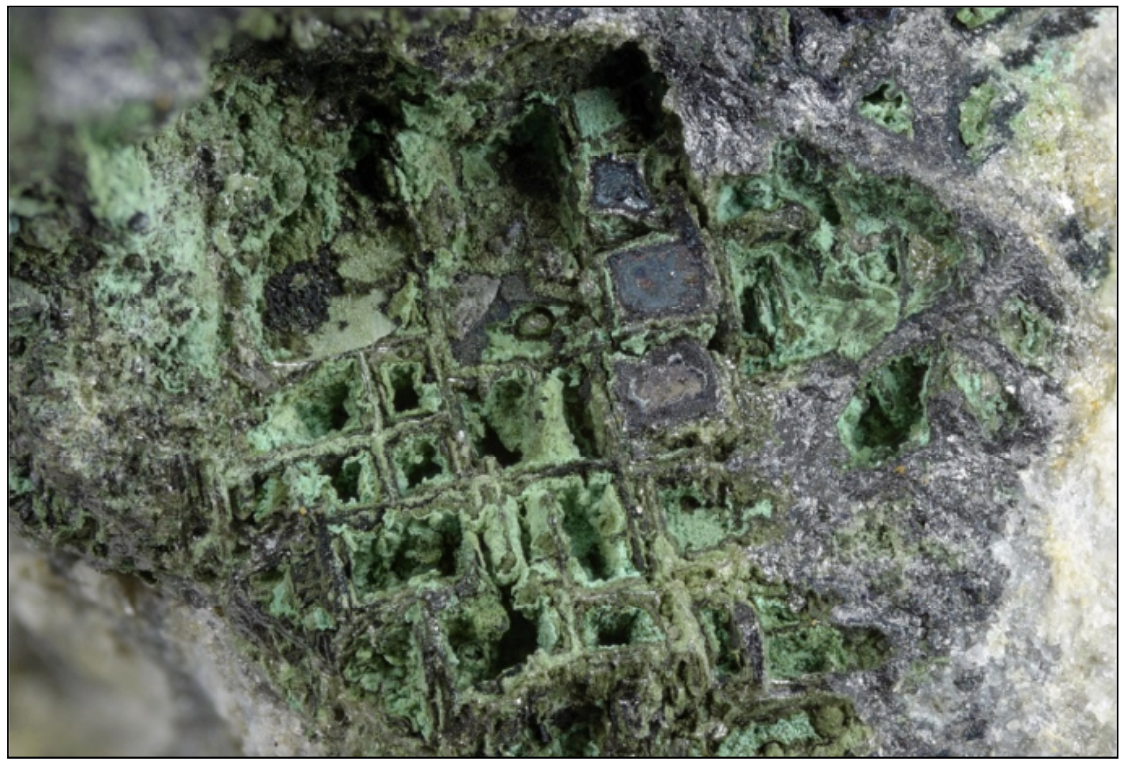

Fig. 18 : Enduit de malachite dans des cavités cubiques formées par disparition de la galène, Masbourg. L = 5,6 mm. Photo P. Chollet. 


\subsection{Plumbojarosite, $\mathrm{Pb}_{0,5} \mathrm{Fe}_{3}\left(\mathrm{SO}_{4}\right)_{2}(\mathrm{OH})_{6}$}

L'analyse par diffraction des rayons $\mathrm{X}$ de petits cristaux, observés dans des plages ocre associées à la galène, montre qu'il s'agit d'un minéral du groupe de la jarosite (Figure 19). Son association à la galène a fait suspecter la plumbojarosite, ce qui a été prouvé par des analyses chimiques qualitatives effectuées par EDS qui indiquent bien la présence majoritaire des éléments $\mathrm{Fe}, \mathrm{S}, \mathrm{O}$ et $\mathrm{Pb}$, avec K minoritaire.

La présence de potassium permet d'identifier un membre intermédiaire de la série jarositeplumbojarosite. Bien que qualitative, l'analyse chimique EDS montre une dominance du plomb par rapport au potassium, ce qui permet d'attribuer le minéral à la plumbojarosite. La dimension des cristaux ne dépasse guère la dizaine de micromètres seulement.

La plumbojarosite est un minéral généralement discret mais sans doute assez fréquent dans les gîtes métallifères belges. Nous l'avons déjà observée notamment à La Roche (Blondieau \& Hatert, 2009), Bure (Blondieau, 2011), Rabotrath (Blondieau et al., 2016), Vielsalm (Blondieau et al., 2017), et Erlenbach.

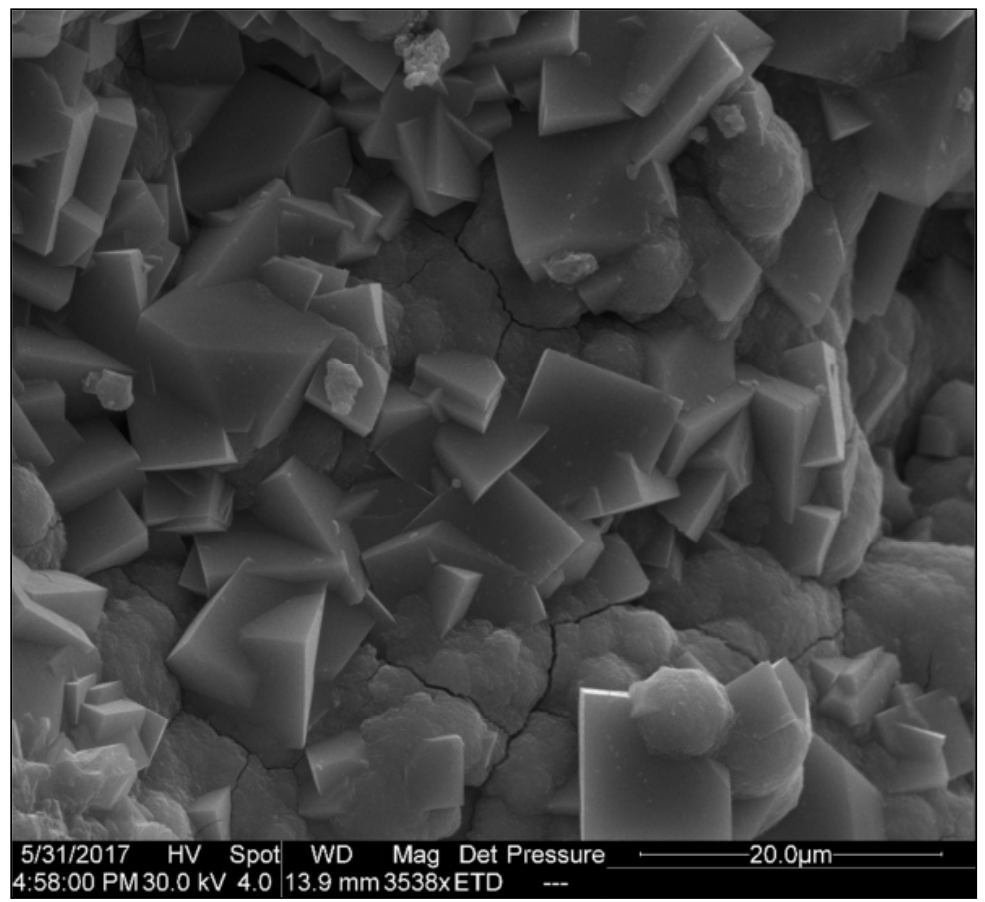

Fig 19 : Plumbojarosite de Masbourg. Échantillon MAS 04. Photographie au microscope électronique à balayage, mode électrons secondaires.

\subsection{Pyrite, $\mathrm{FeS}_{2}$}

La pyrite, rarement observée, forme de petits cubes de maximum un millimètre de côté, en inclusions dans la chalcopyrite (Figure 20), comme c'est le cas dans la mine de cuivre de Stolzembourg, Grand-Duché de Luxembourg. Elle forme plus fréquemment des encroûtements 
Bulletin de la Société Royale des Sciences de Liège, Vol. 86, articles, 2017, p. 169 - 201

pluri-centimétriques qui, examinés sous la loupe, se montrent constitués d'une association de nombreux petits cristaux octaédriques (Figure 21). L'identification est confirmée par diffraction des rayons $\mathrm{X}$, et grâce aux analyses chimiques qualitatives par EDS qui montrent la seule présence des éléments $\mathrm{Fe}$ et $\mathrm{S}$.

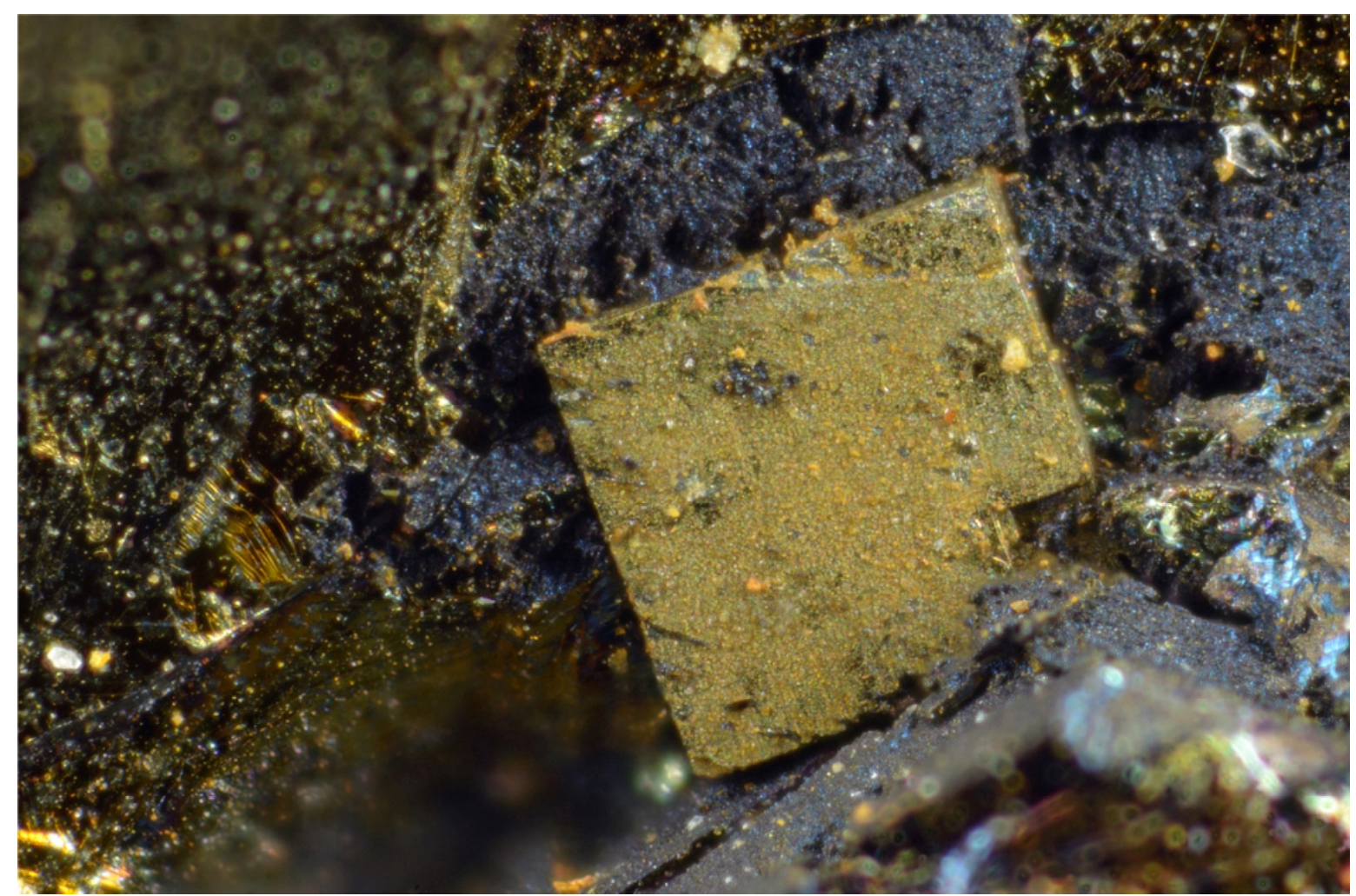

Fig. 20 : Pyrite adoptant l'habitus cubique, en association avec chalcopyrite et covellite, Masbourg. L = 1,2 mm. (Photo P. Chollet). 
Bulletin de la Société Royale des Sciences de Liège, Vol. 86, articles, 2017, p. 169 - 201
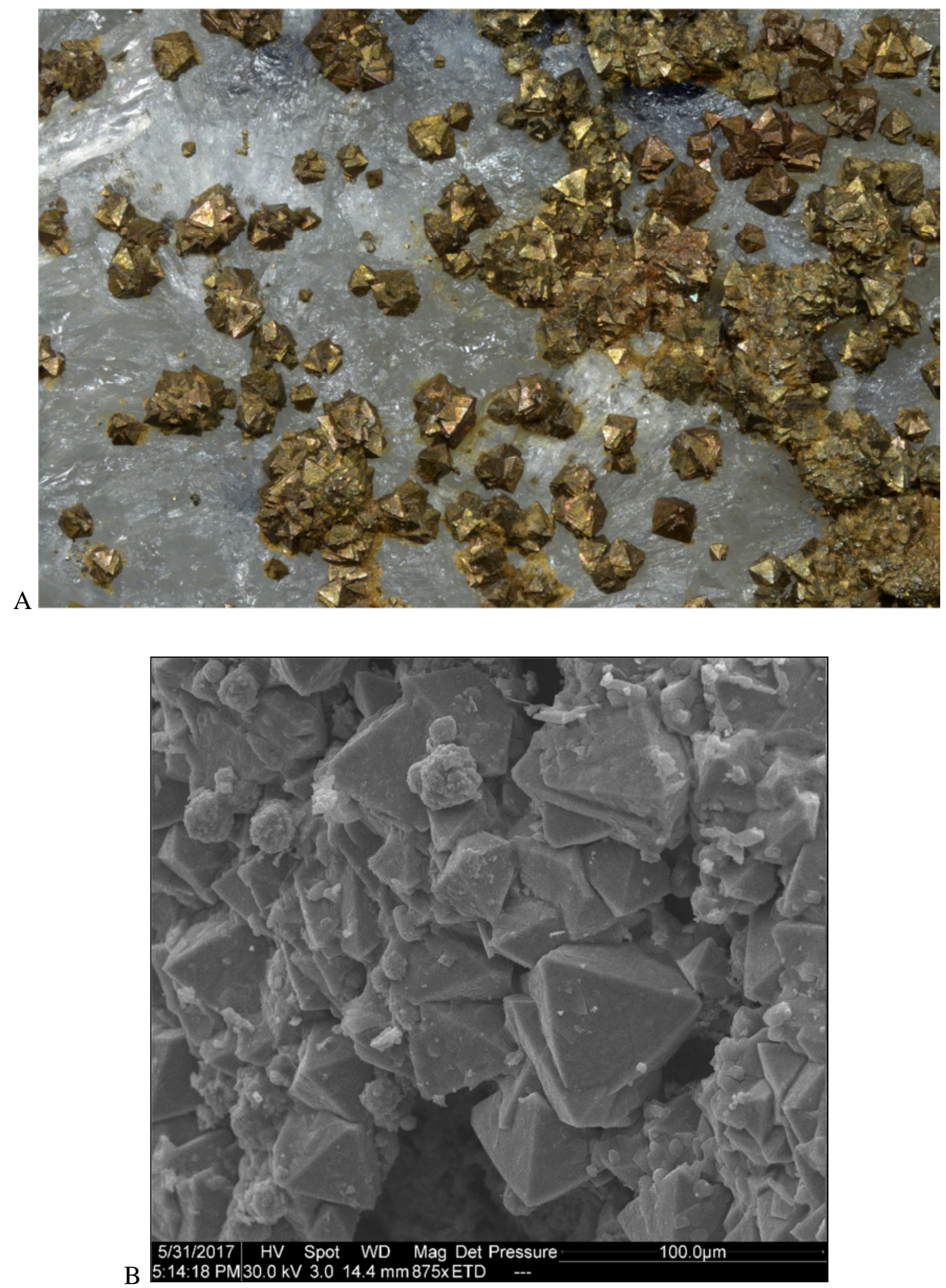

Fig. 21 : Pyrite adoptant l'habitus octaédrique, Masbourg. (A) L = 4 mm, Photo P. Chollet.; (B) Échantillon MAS 02. Photographie au microscope électronique à balayage, mode électrons secondaires. 
Bulletin de la Société Royale des Sciences de Liège, Vol. 86, articles, 2017, p. 169 - 201

\subsection{Quartz, $\mathrm{SiO}_{2}$}

Le quartz laiteux constitue la gangue du filon, mais parfois, on peut observer de petits cristaux incolores à laiteux, avec la forme du prisme assez peu développée (Figure 22). Généralement, la taille des cristaux ne dépasse pas quelques millimètres.

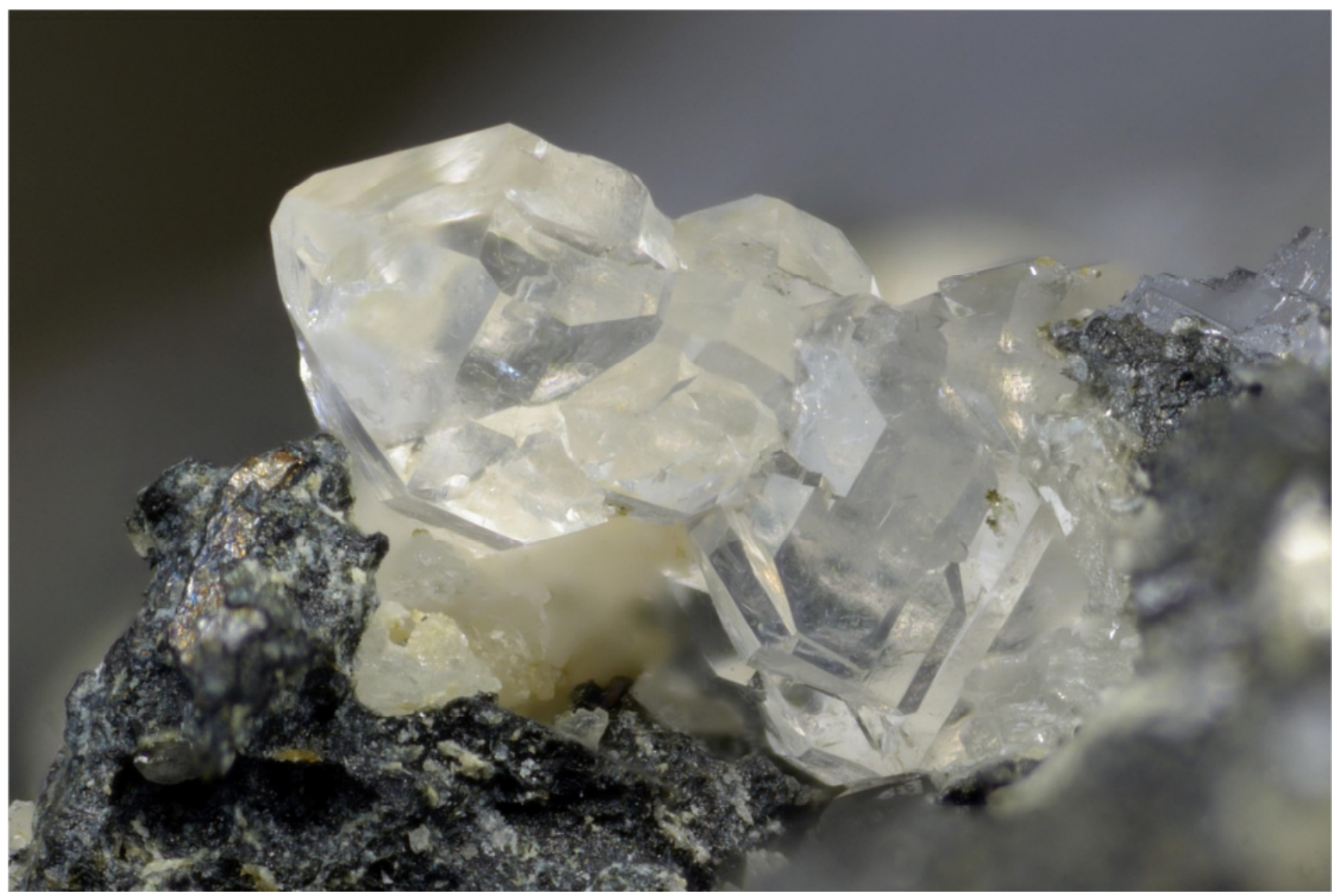

Fig. 22 : Quartz, Masbourg. $\mathrm{L}=1,85 \mathrm{~mm}$. (Photo P. Chollet).

\subsection{Soufre, $S$}

De très petits cristaux jaune pâle sont observés dans des blocs de quartz à galène corrodée et devenue "spongieuse". L'analyse par diffraction des rayons $X$ indique qu'il s'agit de soufre (Figure 23). Le soufre est une espèce secondaire fréquente qui se forme dans les tas de minerais sulfurés soumis à l'altération météorique.

\subsection{Sphalérite, $(\mathrm{Zn}, \mathrm{Fe}) \mathrm{S}$}

Des petites plages brun miel millimétriques à centimétriques, associées à la galène et à la chalcopyrite, sont visuellement déterminées comme de la sphalérite, ce qui est confirmé par diffraction des rayons X. Le minéral, très peu spectaculaire, est fort peu fréquent à Masbourg ; il n'a été observé que sur quelques échantillons. 
Bulletin de la Société Royale des Sciences de Liège, Vol. 86, articles, 2017, p. 169 - 201
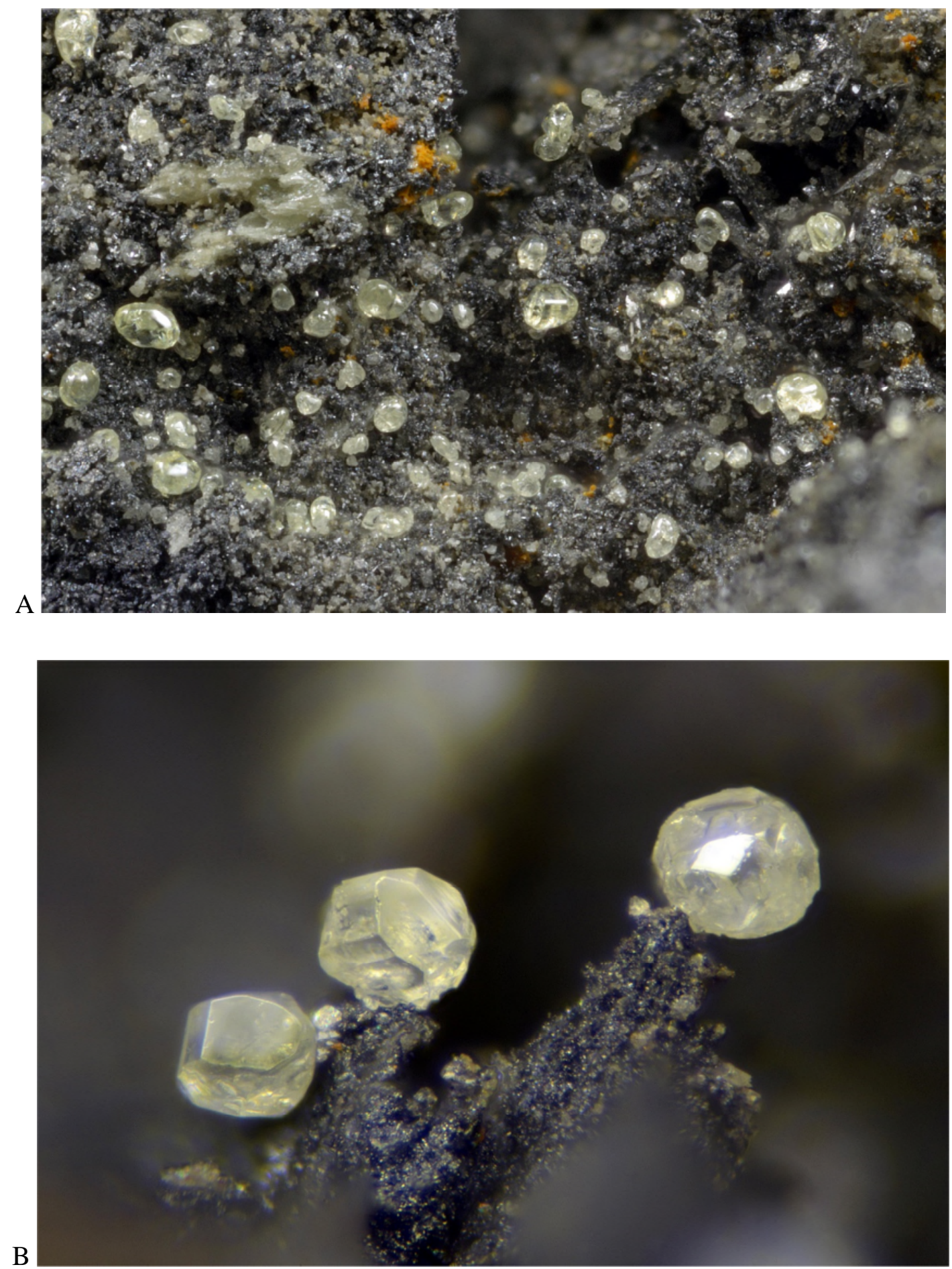

Fig. 23 : Petits cristaux de soufre, Masbourg. (A) $\mathrm{L}=2,1 \mathrm{~mm}$; (B) $\mathrm{L}=0,55 \mathrm{~mm}$; (A) et (B) (Photos P. Chollet). 
Bulletin de la Société Royale des Sciences de Liège, Vol. 86, articles, 2017, p. 169 - 201

\subsection{Susannite, $\mathrm{Pb}_{4}\left(\mathrm{SO}_{4}\right)\left(\mathrm{CO}_{3}\right)_{3}(\mathrm{OH})_{2}$}

Des petits cristaux blanchâtres d'une centaine de microns de diamètre, en tablettes hexagonales épaisses, sont parfois observés dans les parties riches en brochantite et linarite de certains blocs de galène corrodée (Figure 24).

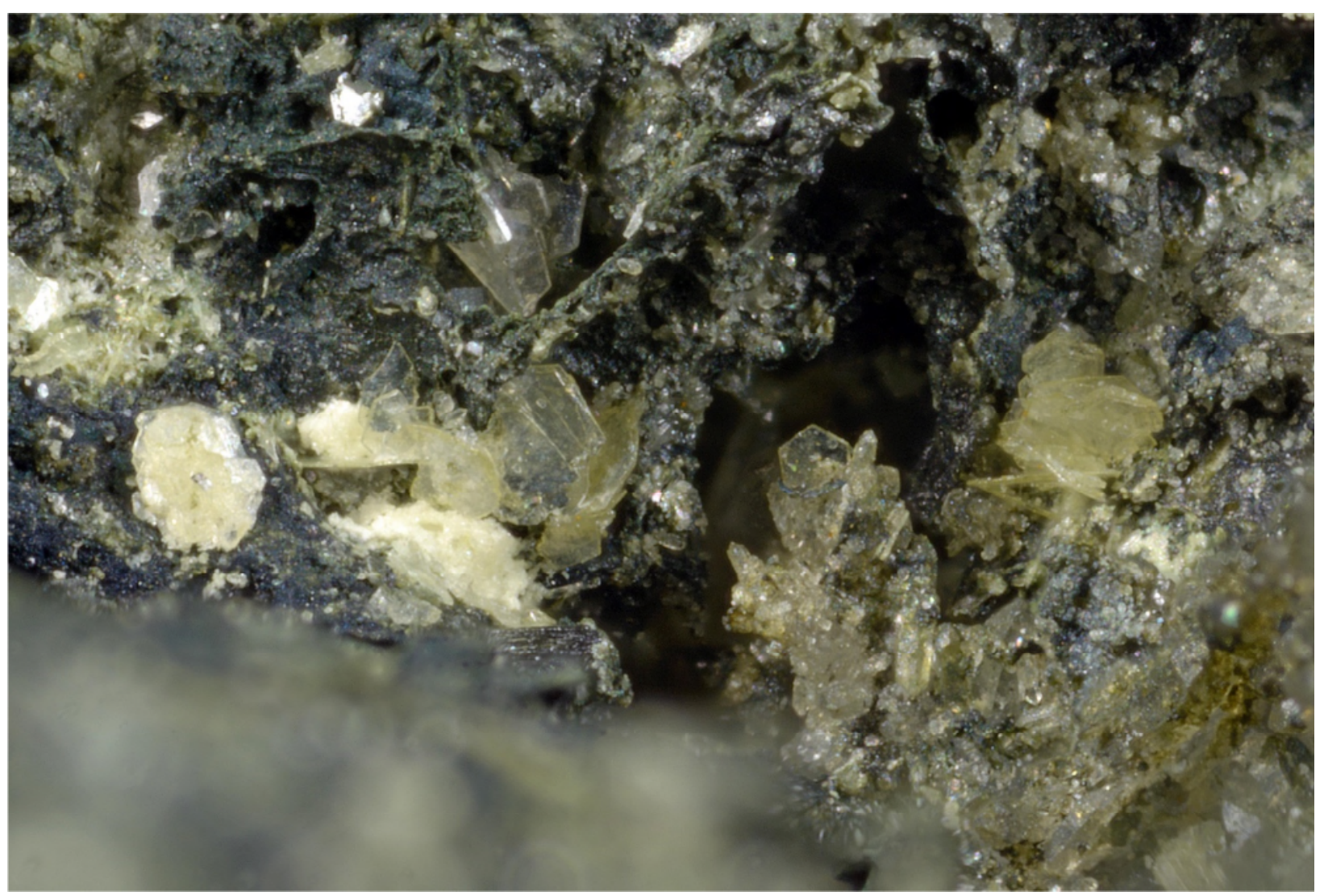

Fig. 24 : Susannite de Masbourg. Un petit cristal de forme hexagonale est bien visible au milieu de la petite cavité. $\mathrm{L}=1,8 \mathrm{~mm}$. (Photo P. Chollet).

Les analyses chimiques qualitatives EDS indiquent la présence des éléments $\mathrm{Pb}, \mathrm{C}, \mathrm{O}$ et $\mathrm{S}$. Cette composition chimique et la forme cristalline suggèrent la leadhillite ou la susannite. Ces deux espèces sont très proches d'un point de vue cristallochimique; Bindi \& Menchetti (2005) ont d'ailleurs montré une transformation réversible et progressive de la leadhillite en susannite dans la fourchette de température $50-82{ }^{\circ} \mathrm{C}$. Un monocristal de Masbourg a été soumis au diffractomètre de rayons $\mathrm{X}$ à 4 cercles, ce qui a permis de déterminer les paramètres de maille : $a=9,038(3)$ et $c=11,570(2) \AA$. Ces paramètres sont légèrement plus proches de ceux de la susannite, semblant indiquer qu'il s'agit de ce minéral, plutôt que de la leadhillite. Toutefois, les paramètres de maille des deux espèces sont très proches (Bindi \& Menchetti, 2005 ; Steele et al., 1999) ; l'identification est délicate. 
Bulletin de la Société Royale des Sciences de Liège, Vol. 86, articles, 2017, p. 169 - 201

Sur le matériel de Masbourg, on pourrait, visuellement, confondre la susannite avec des cristaux de dickite, mais ces derniers sont extrêmement minces, contrairement à ceux de susannite qui sont très épais. Cette caractéristique est d'ailleurs bien mise en évidence par les photos prises en microscopie électronique à balayage (Figure 25). Ces photos permettent aussi d'observer des faces cristallines sur les cristaux de susannite de Masbourg ; ils correspondent à la combinaison $\{10 \overline{1} 0\}\{20 \overline{2} 1\}\{0001\}$ (Figures 25B, C et D).

La susannite est une espèce secondaire très rare qui n'avait pas encore été signalée en Belgique.

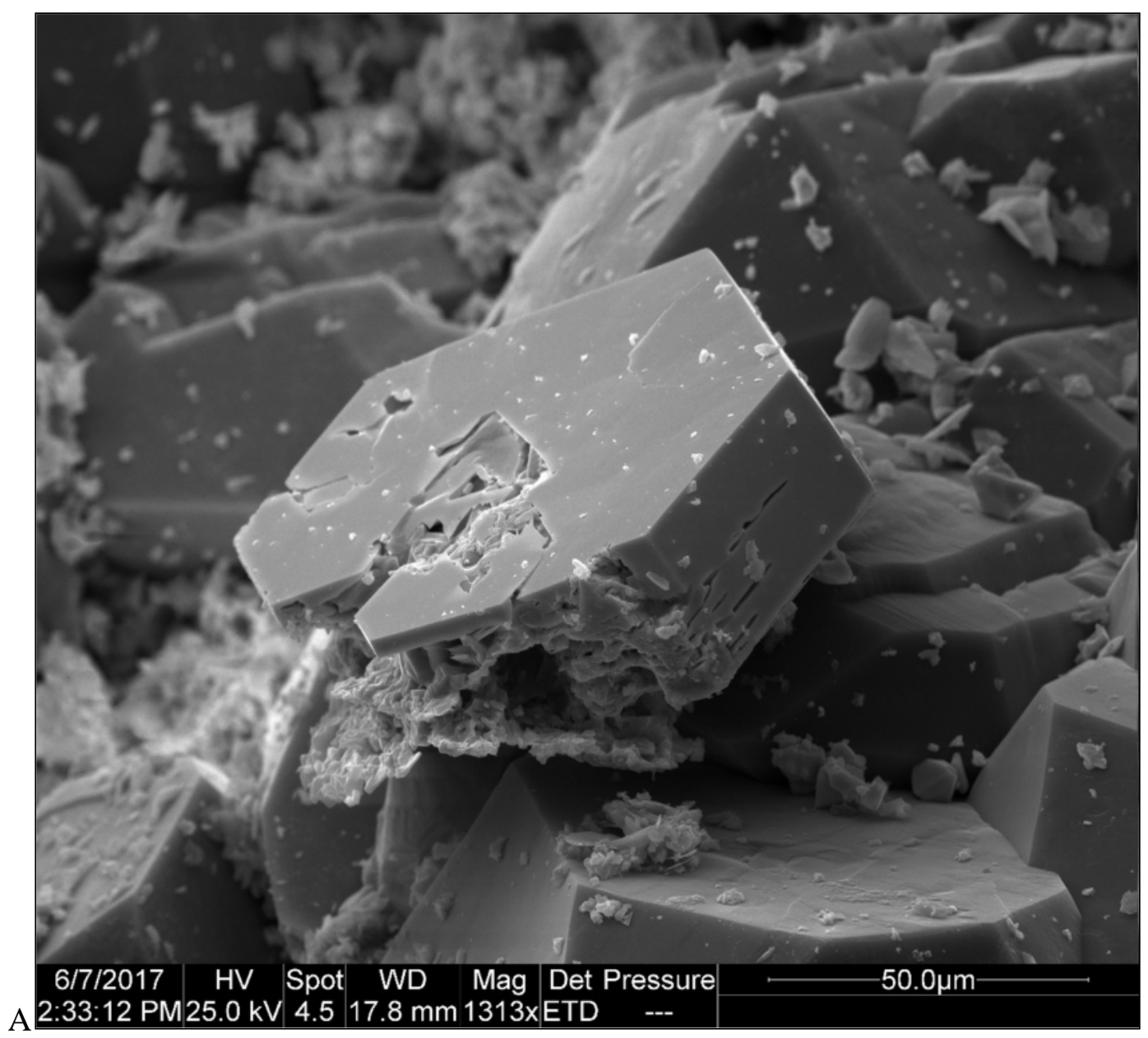


Bulletin de la Société Royale des Sciences de Liège, Vol. 86, articles, 2017, p. 169 - 201
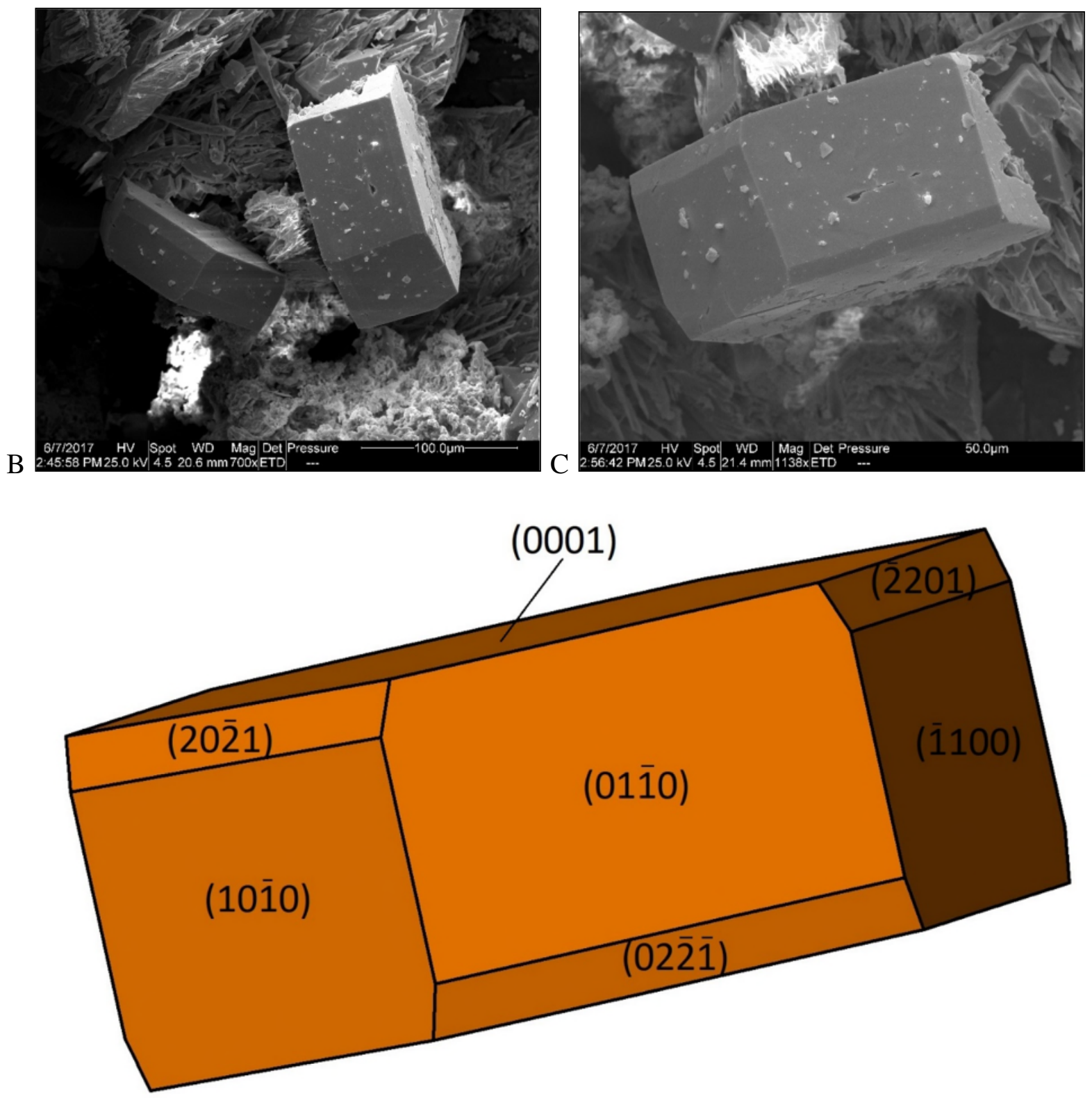

$\mathrm{D}$

Fig. 25 : Susannite de Masbourg. (A) Échantillon MAS 07 ; (B) et (C) Échantillon MAS 08. Photographies au microscope électronique à balayage, mode électrons secondaires.

(D) Morphologie du minéral, montrant la combinaison $\{10 \overline{1} 0\}\{20 \overline{2} 1\}\{0001\}$.

\subsection{Todorokite, $(\mathrm{Na}, \mathrm{Ca}, \mathrm{K}, \mathrm{Ba}, \mathrm{Sr})_{1-\mathrm{x}}(\mathrm{Mn}, \mathrm{Mg}, \mathrm{Al})_{6} \mathrm{O}_{12} \cdot 3-4 \mathrm{H}_{2} \mathrm{O}$}

Sur les échantillons présentant des pseudomorphoses ayant conservé une forme rhomboédrique de dolomite (Figure 14), on peut observer un minéral en petites masses brunes à violacées, d'aspect spongieux (Figure 26). Visuellement déterminé comme todorokite, l'identification est confirmée par diffraction des rayons $\mathrm{X}$. 


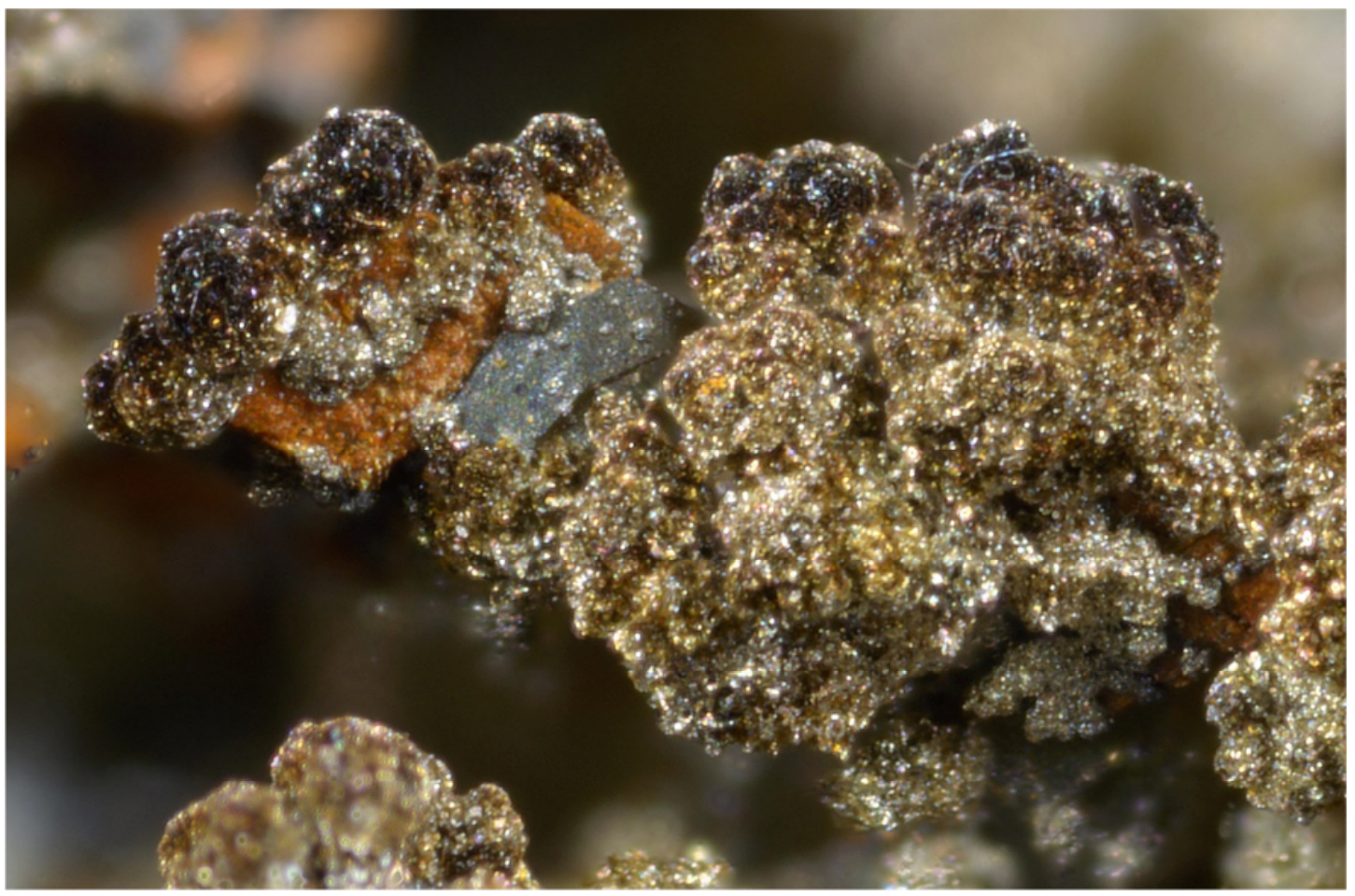

Fig. 26 : Todorokite de Masbourg. $\mathrm{L}=1,35 \mathrm{~mm}$. (Photo P. Chollet).

\section{Conclusions}

Le petit gisement plombifère de Masbourg, à gangue quartzeuse et d'importance secondaire du point de vue des tonnages de minerai produits, présente une minéralogie peu commune pour la Belgique, un pays où l'on a exploité de gros gisements de zinc et de plomb dans des encaissants carbonatés. En effet, deux espèces nouvelles pour notre pays y sont recensées : la calédonite et la susannite.

La mise en place des minéralisations de Masbourg pourrait être schématisée de la manière suivante :

1) Ouverture de failles dans les roches du dévonien inférieur.

2) Des solutions déposent des cristaux de quartz incolore sur les épontes. Les failles doivent travailler à plusieurs reprises puisqu' on retrouve du minerai bréchique (Figure 3) ainsi que parfois de petits miroirs de faille. Des petits cristaux de quartz recouvrent tous les matériaux tombés par gravité dans la faille.

3) Localement, un fin liseré de pyrite vient se superposer aux cristaux de quartz.

4) Minéralisation en galène sur la pyrite ou, plus fréquemment, directement sur les cristaux de quartz.

5) Dépôt d'un peu de chalcopyrite, surtout dans les parties les plus larges du filon. 
Bulletin de la Société Royale des Sciences de Liège, Vol. 86, articles, 2017, p. 169 - 201

6) Oxydation et formation de cérusite dans les fissures de la galène mais aussi de l'encaissant. Dépôt d'« argiles blanches » (dickite) résultant probablement de phénomènes d'altération sous climat tropical, comme c'est le cas pour les gros dépôts de kaolin exploités en Ardenne.

Le Tableau 1 reprend la liste complète des 24 espèces minérales décrites à Masbourg.

Tableau 1 : Espèces minérales de Masbourg. Les espèces en «gras » sont nouvelles pour la Belgique.

\begin{tabular}{|c|c|}
\hline Minéral & Formule chimique idéale \\
\hline Anglésite & $\mathrm{PbSO}_{4}$ \\
\hline Aragonite & $\mathrm{CaCO}_{3}$ \\
\hline Aurichalcite & $(\mathrm{Zn}, \mathrm{Cu})_{5}\left(\mathrm{CO}_{3}\right)_{2}(\mathrm{OH})_{6}$ \\
\hline Brochantite & $\mathrm{Cu}_{4}\left(\mathrm{SO}_{4}\right)(\mathrm{OH})_{6}$ \\
\hline Calcite & $\mathrm{CaCO}_{3}$ \\
\hline Calédonite & $\mathrm{Cu}_{2} \mathrm{~Pb}_{5}\left(\mathrm{SO}_{4}\right)_{3}\left(\mathrm{CO}_{3}\right)(\mathrm{OH})_{6}$ \\
\hline Cérusite & $\mathrm{PbCO}_{3}$ \\
\hline Chalcopyrite & $\mathrm{CuFeS}_{2}$ \\
\hline Chamosite & $\left(\mathrm{Fe}^{2+}, \mathrm{Mg}, \mathrm{Al}, \mathrm{Fe}^{3+}\right)_{6}(\mathrm{Si}, \mathrm{Al})_{4} \mathrm{O}_{10}(\mathrm{OH}, \mathrm{O})_{8}$ \\
\hline Covelline & $\mathrm{CuS}$ \\
\hline Cuprite & $\mathrm{Cu}_{2} \mathrm{O}$ \\
\hline Dickite & $\mathrm{Al}_{2} \mathrm{Si}_{2} \mathrm{O}_{5}(\mathrm{OH})_{4}$ \\
\hline Dolomite & $\mathrm{CaMg}\left(\mathrm{CO}_{3}\right)_{2}$ \\
\hline Galène & $\mathrm{PbS}$ \\
\hline Hydrozincite & $\mathrm{Zn}_{5}\left(\mathrm{CO}_{3}\right)_{2}(\mathrm{OH})_{6}$ \\
\hline Linarite & $\mathrm{CuPb}\left(\mathrm{SO}_{4}\right)(\mathrm{OH})_{2}$ \\
\hline Malachite & $\mathrm{Cu}_{2}\left(\mathrm{CO}_{3}\right)(\mathrm{OH})_{2}$ \\
\hline Plumbojarosite & $\mathrm{Pb}_{0,5} \mathrm{Fe}_{3}\left(\mathrm{SO}_{4}\right)_{2}(\mathrm{OH})_{6}$ \\
\hline Pyrite & $\mathrm{FeS}_{2}$ \\
\hline Quartz & $\mathrm{SiO}_{2}$ \\
\hline Soufre & S \\
\hline Sphalérite & $(\mathrm{Zn}, \mathrm{Fe}) \mathrm{S}$ \\
\hline Susannite & $\mathrm{Pb}_{4}\left(\mathrm{SO}_{4}\right)\left(\mathrm{CO}_{3}\right)_{3}(\mathrm{OH})_{2}$ \\
\hline Todorokite & $(\mathrm{Na}, \mathrm{Ca}, \mathrm{K}, \mathrm{Ba}, \mathrm{Sr})_{1-\mathrm{x}}(\mathrm{Mn}, \mathrm{Mg}, \mathrm{Al})_{6} \mathrm{O}_{12} \cdot 3-4 \mathrm{H}_{2} \mathrm{O}$ \\
\hline
\end{tabular}

\section{Remerciements :}

Nous souhaitons remercier Fabrice Dal Bo pour l'aide importante apportée lors de l'utilisation des diffractomètres au laboratoire, ainsi que pour l'aide précieuse qu'il nous a toujours apportée 
Bulletin de la Société Royale des Sciences de Liège, Vol. 86, articles, 2017, p. 169 - 201

pour le dépouillement des résultats d'analyse et tout particulièrement pour ceux des cristaux de susannite. Nos remerciements vont aussi à Pascal Chollet qui a réalisé les magnifiques photos de nos très petits minéraux, à Philippe Compère pour son aide lors de l'utilisation du microscope électronique à balayage, ainsi qu'à Monsieur Hicham Naji qui nous a fourni des scans de nombreux documents d'archive conservés au SPW (Service Public de Wallonie) à Jambes.

\section{Références}

Asselberghs, E. (1946). L'éodévonien de l'Ardenne et des régions voisines. Mémoire de l'Institut de Géologie de l'Université de Louvain, 16, 598 p.

Bindi L. \& Menchetti S. (2005) Structural changes accompanying the phase transformation between leadhillite and susannite : A structural study by means of in situ high-temperature singlecrystal X-ray diffraction. American mineralogist, 90, 1641-1647

Blockmans S. \& Dumoulin V. (2017). Planchette 59/7-8 de la nouvelle Carte géologique de la Wallonie. Sous presse.

Blondieau M. \& Hatert F. (2009). Minéralogie de l'ancienne mine de plomb de La Roche-enArdenne (Belgique). Le Règne Minéral, 88, 5-20.

Blondieau M. (2011). Anciens travaux de recherches pour plomb à Bure : Historique, localisation et minéralogie. De la Meuse à l'Ardenne, 43, 61-93.

Blondieau, M. \& Polrot, F. (2011). Les travaux miniers de Schimper, siège sud de la mine du Bleyberg (Plombières, Belgique) : plomb, zinc mais aussi argent. Geological Survey of Belgium, Professional Paper, 310, 57 pages.

Blondieau M., Puccio S., Compère P. \& Hatert F. (2016). Minéralogie de l'ancienne mine plombzinc de Rabotrath (Lontzen, Belgique). Bulletin de la Société Royale des Sciences de Liège, $\mathbf{8 5}$, $30-55$.

Blondieau M., Puccio S., Compère P. \& Hatert F. (2017). Données nouvelles sur quelques espèces minérales de Vielsalm et de Salmchâteau (Province de Luxembourg, Belgique). Bulletin de la Société Royale des Sciences de Liège, 86, 1-48.

Bockholz, G. (1921). Note descriptive de la mine de Masbourg. SPW - $N^{\circ} 19210204$. Dejonghe L. \& Hance L. (2001). Notice explicative de la carte géologique de Wallonie 1:2500, 60/1-2 - Champlon, La Roche-en-Ardenne. 44 pages. 
Bulletin de la Société Royale des Sciences de Liège, Vol. 86, articles, 2017, p. 169 - 201

Hatert F., Deliens M., Fransolet A.-M. \& Van Der Meersche E. (2002). Les minéraux de Belgique, deuxième édition. 304 pages.

Poncelet Ing. (1856 et 1861). État d'exploitation de la mine de Masbourg (Province de Luxembourg) - Archives de l'État à Arlon, Inventaire des archives de l'administration provinciale du Luxembourg. Série des établissements dangereux, insalubres ou incommodes : mines, minières et carrières (1817)(1830-1990)/Magali Denoncin et Vincent Pirlot. BEA0521_702127_701177_FRE, Liasse n ${ }^{\circ}$ 58, Masbourg.

Seeliger A. (1852). Plan de demande de concession. $S P W-N^{\circ} 18521019$.

Sonnet, P. (2017). Inventaire géochimique des ressources métallifères de la Wallonie. http://geologie.wallonie.be/home/thematiques-sous-sol/inventaire-geochimique.html (accès juin 2017) 